\title{
Weak convergence of SFDEs driven by fractional Brownian motion with irregular coefficients
}

\author{
Yongqiang Suo ${ }^{a)}$, Chenggui Yuan ${ }^{a)}$, Shao-Qin Zhang ${ }^{b)}$ \\ a) Department of Mathematics, Swansea University, Bay campus, SA1 8EN, UK \\ 971001@swansea.ac.uk, C.Yuan@swansea.ac.uk \\ b) Central University of Finance and Economics, Beijing 100081, China \\ zhangsq@cufe.edu.cn
}

\begin{abstract}
In this paper, we investigate weak existence and uniqueness of solutions and weak convergence of Euler-Maruyama scheme to stochastic functional differential equations with Hölder continuous drift driven by fractional Brownian motion with Hurst index $H \in(1 / 2,1)$. The methods used in this paper are Girsanov's transformation and the property of the corresponding reference stochastic differential equations.
\end{abstract}

AMS Subject Classification: 60F10, 60H10, 34K26.

Keywords: Weak solution, weak convergence, Hölder continuity drift, fractional Brownian motion

\section{Introduction}

The fractional Brownian motion (fBM) appears naturally in modeling stochastic systems with long-range dependence phenomena in applications. Fractional Brownian motions with Hurst parameter $H \neq 1 / 2$ are neither Markov processes nor (weak) semimartingales, which makes the study of stochastic differential equations (SDEs) driven by fBMs complicated. The existence and uniqueness of solutions to fractional equations have received much attention. [13] obtained existence and uniqueness of solutions to SDEs driven by fBMs with Hurst parameter $H \in\left(\frac{1}{2}, 1\right)$ by using Young integrals (see [30]) and $p$-variation estimate; [3] derived the existence and uniqueness result for $H \in\left(\frac{1}{4}, \frac{1}{2}\right)$ through the same rough-type arguments in [13]; [25] studied SDEs driven by fBMs by using fractional calculus developed in [31]. For more results on existence and uniqueness of solutions to SDEs driven by fBMs, we refer to $[2,8,9,12,17,24]$ for instance. Stochastic functional differential equations (SFDEs) are 
also used to characterise stochastic systems with memory effects. For the existence and uniqueness of solutions for SFDEs with regular coefficients, one can consult to [6, 19, 21]. In recent years, SDEs driven by $\mathrm{fBM}$ with irregular coefficients have received much attention, e.g. $[5,9]$. However, for fractional SFDEs with irregular coefficients, even the weak existence and uniqueness results are not well studied. So, we first study the weak existence and uniqueness for SFDEs driven by fBMs (see Theorem 3.1 below), based on which we shall give a weak convergence result on the weak solution of fractional SFDEs with irregular drift (see Theorem 3.2). By using the associated Kolmogorov equations, SDEs with irregular coefficients driven by Brownian motion or Lévy noise are intensively studied. However, this powerful tool seems hard to be applied to fractional SDEs. To study weak solutions, we adopt Girsanov's transformation. In the case of SDEs driven by fBMs, it involves fractional calculus to ensure that Girsanov's transformation can be applied, and the related estimates are nontrivial for the irregular drift with memory.

Weak error for the Euler scheme approximation of SDEs driven by Brownian motion with irregular coefficients are intensively studied recently, e.g. [10, 11, 22] and references therein. In [10, 11], test functions of the weak convergence are regular. In [22], the authors get weak convergence for test function without regularity. There is a few literature on the convergence of numerical schemes for SDEs driven by fBMs, e.g. [7, 15, 16, 18, 19, 20, 28]. Recently, [1] developed a perturbation argument to investigate the weak convergence of SFDEs with irregular coefficients by using Girsanov's transformation. Based on our weak existence and uniqueness result, we investigate the weak convergence of truncated Euler-Maruyama (EM) scheme for SFDEs driven by fBMs by using a test function without assuming regularity. The drift depends on past, and it is also irregular. The exponential integrability of functionals of the segment process (see the beginning of Section 3) studied in our work involves fractional calculus, which is more complicated than those of SFDEs driven by Brownian motion. Explicit convergence order is given for the numerical scheme, and the main ingredient is giving exact estimates for fractional derivatives of functionals of the segment process truncated by gridpoints, see Lemma 5.2.

The paper is organised as follows: Section 2 is devoted to the preliminaries containing fractional calculus and some properties of fBM; in Section 3, we state our main results on weak existence and uniqueness and numerical approximation; proofs are provided in Section 4 and Section 5.

\section{Preliminaries}

\subsection{Fractional integrals and derivatives}

In this subsection, we recall some basic facts about fractional integrals and derivatives, for more details, see $[23,27]$.

Let $a, b \in \mathbb{R}$ with $a<b$. For $f \in L^{1}(a, b)$ and $\alpha>0$, the left-sided fractional Riemann- 
Liouville integral of order $\alpha$ of $f$ on $[a, b]$ is given by

$$
I_{a+}^{\alpha} f=\frac{1}{\Gamma(\alpha)} \int_{a}^{x} \frac{f(y)}{(x-y)^{1-\alpha}} \mathrm{d} y,
$$

where $x \in(a, b)$ a.e. $(-1)^{-\alpha}=\mathrm{e}^{-i \alpha \pi}, \Gamma$ denotes the Euler function. If $\alpha=n \in \mathbb{N}$, this definition coincides with the $n$-order iterated integrals of $f$. By the definition, we have the first composition formula

$$
I_{a+}^{\alpha}\left(I_{a+}^{\beta} f\right)=I_{a+}^{\alpha+\beta} f .
$$

Fractional differentiation may be introduced as an inverse operation. Let $\alpha \in(0,1)$ and $p \geq 1$. If $f \in I_{a+}^{\alpha}\left(L^{p}([a, b], \mathbb{R})\right)$, the function $\phi$ satisfying $f=I_{a+}^{\alpha} \phi$ is unique in $L^{p}([a, b], \mathbb{R})$ and it coincides with the left sided Riemann-Liouville derivative of $f$ of order $\alpha$ given by

$$
D_{a+}^{\alpha} f(x)=\frac{1}{\Gamma(1-\alpha)} \frac{\mathrm{d}}{\mathrm{d} x} \int_{a}^{x} \frac{f(y)}{(x-y)^{\alpha}} \mathrm{d} y .
$$

The corresponding Weyl representation reads as follows

$$
D_{a+}^{\alpha} f(x)=\frac{1}{\Gamma(1-\alpha)}\left(\frac{f(x)}{(x-a)^{\alpha}}+\alpha \int_{a}^{x} \frac{f(x)-f(y)}{(x-y)^{1+\alpha}} \mathrm{d} y\right),
$$

where the convergence of the integrals at the singularity $y=x$ holds pointwise for almost all $x$ if $p=1$ and in the $L^{p}$ sense if $p>1$. By the construction, we have

$$
I_{a+}^{\alpha}\left(D_{a+}^{\alpha} f\right)=f, \quad f \in I_{a+}^{\alpha}\left(L^{p}([a, b], \mathbb{R})\right),
$$

and moreover it holds the second composition formula

$$
D_{a+}^{\alpha}\left(D_{a+}^{\beta} f\right)=D_{a+}^{\alpha+\beta} f, \quad f \in I_{a+}^{\alpha+\beta}\left(L^{1}([a, b], \mathbb{R})\right) .
$$

\section{$2.2 \quad$ Fractional Brownian motion}

To make the content self-contained, we first recall some basic facts about the stochastic calculus of variations with respect to the fBM with Hurst parameter $H \in\left(\frac{1}{2}, 1\right)$. We refer the reader to [4] for further details.

Fixe $T>0$. The $d$-dimensional fBm $B^{H}=\left\{B^{H}(t), t \in[0, T]\right\}$ with Hurst parameter $H$ on the complete probability space $(\Omega, \mathscr{F}, \mathbb{P})$ can be defined as the centered Gauss process with covariance function

$$
\mathbb{E}\left(B^{H}(t) B^{H}(s)\right)=R_{H}(t, s)=\frac{1}{2}\left(t^{2 H}+s^{2 H}-|t-s|^{2 H}\right) .
$$

In particular, if $H=\frac{1}{2}, B^{H}$ is a Brownian motion. Besides,

$$
\mathbb{E}\left|B^{H}(t)-B^{H}(s)\right|^{p}=\mathbb{E}\left|B^{H}(t-s)\right|^{p}=|t-s|^{p H} \mathbb{E}\left|B^{H}(1)\right|^{p} \leq C(p)|t-s|^{p H}, p \geq 1 .
$$


Then it follows from the Kolmogorov continuity theorem that $B^{H}$ has $\beta$-Hölder continuous paths, where $\beta \in(0, H)$. For each $t \in[0, T]$, we denote by $\mathscr{F}_{t}$ the $\sigma$-algebra generated by $\left\{B^{H}(s): s \in[0, t]\right\}$ and the $\mathbb{P}$-null sets.

We denote by $\mathscr{E}$ the set of step functions on $[0, T]$. Let $\mathscr{H}$ be the Hilbert space defined as the closure of $\mathscr{E}$ with respect to the scalar product

$$
\left\langle\left(I_{\left[0, t_{1}\right]}, \cdots, I_{\left[0, t_{d}\right]}\right),\left(I_{\left[0, s_{1}\right]}, \cdots, I_{\left[0, s_{d}\right]}\right)\right\rangle_{\mathscr{C}}=\sum_{i=1}^{d} R_{H}\left(t_{i}, s_{i}\right) .
$$

The mapping $I_{\left[0, t_{1}\right] \times \cdots \times I_{\left[0, t_{d}\right]}} \mapsto\left(B_{t_{1}}^{H, 1}, \cdots, B_{t_{d}}^{H, d}\right)$ can be extended to an isometry between $\mathscr{H}$ and the Gauss space $\mathscr{H}_{1}$ spanned by $B^{H}$. Denote this isometry by $\phi \mapsto B^{H}(\phi)$. On the other hand, from [4], we know the covariance kernel $R_{H}(t, s)$ can be written as

$$
R_{H}(t, s)=\int_{0}^{t \wedge s} K_{H}(t, r) K_{H}(s, r) \mathrm{d} r
$$

where $K_{H}$ is a square integrable kernel given by

$$
K_{H}(t, s)=\Gamma\left(H+\frac{1}{2}\right)^{-1}(t-s)^{H-\frac{1}{2}} F\left(H-\frac{1}{2}, \frac{1}{2}-H, H+\frac{1}{2}, 1-\frac{t}{s}\right),
$$

in which $F(\cdot, \cdot, \cdot, \cdot)$ is Gauss's hypergeometric function (see [4]).

Define the linear operator $K_{H}^{*}: \mathscr{E} \rightarrow L^{2}\left([0, T], \mathbb{R}^{d}\right)$ as follows

$$
\left(K_{H}^{*} \phi\right)(s)=K_{H}(T, s) \phi(s)+\int_{s}^{T}(\phi(r)-\phi(s)) \frac{\partial K_{H}}{\partial r}(r, s) \mathrm{d} r .
$$

Reformulating the above equality as follows:

$$
\left(K_{H}^{*} \phi\right)(s)=\int_{s}^{T} \phi(r) \frac{\partial K_{H}}{\partial r}(r, s) \mathrm{d} r .
$$

It can be shown that for all $\phi, \psi \in \mathscr{E}$,

$$
\left\langle K_{H}^{*} \phi, K_{H}^{*} \psi\right\rangle_{L^{2}\left([0, T], \mathbb{R}^{d}\right)}=\langle\phi, \psi\rangle_{\mathscr{H}},
$$

and therefore $K_{H}^{*}$ is an isometry between $\mathscr{H}$ and $L^{2}\left([0, T], \mathbb{R}^{d}\right)$. Consequently, $B^{H}$ has the following integral representation

$$
B^{H}(t)=\int_{0}^{t} K_{H}(t, s) \mathrm{d} B(s)
$$

where $\left\{B(t):=B^{H}\left(\left(K_{H}^{*}\right)^{-1} I_{[0, t]}\right)\right\}$ is a standard Brownian motion.

According to [4], the operator $K_{H}: L^{2}\left([0, T], \mathbb{R}^{d}\right) \rightarrow I_{0+}^{H+\frac{1}{2}}\left(L^{2}\left([0, T], \mathbb{R}^{d}\right)\right)$ associated with the kernel $K_{H}(\cdot, \cdot)$ is defined as follows

$$
\left(K_{H} f^{i}\right)(t)=\int_{0}^{t} K_{H}(t, s) f^{i}(s) \mathrm{d} s, \quad i=1, \cdots, d .
$$


It can be proved that $K_{H}$ is an isomorphism. Moreover, for each $f \in L^{2}\left([0, T], \mathbb{R}^{d}\right)$,

$$
\left(K_{H} f\right)(s)=I_{0+}^{1} s^{H-1 / 2} I_{0+}^{H-1 / 2} s^{1 / 2-H} f, H>\frac{1}{2} .
$$

Consequently, for each $h \in I_{0+}^{H+1 / 2}\left(L^{2}\left([0, T], \mathbb{R}^{d}\right)\right)$, the inverse operator $K_{H}^{-1}$ is of the form

$$
\left(K_{H}^{-1} h\right)(s)=s^{H-1 / 2} D_{0+}^{H-1 / 2} s^{1 / 2-H} h^{\prime}, H>\frac{1}{2} .
$$

We conclude this section by introducing the following Fernique-type lemma (see [14, 26]) and some notation for future use.

Lemma 2.1. Let $T>0,1 / 2<\beta<H<1$. Then for any $\alpha<\frac{1}{2 T}$,

$$
\mathbb{E} \exp \left\{\alpha\left\|B^{H}\right\|_{0, T, \infty}^{2}\right\}<\infty
$$

and for any $\alpha<1 /\left(128(2 T)^{2(H-\beta)}\right)$,

$$
\mathbb{E}\left[\exp \left(\alpha\left\|B^{H}\right\|_{0, T, \beta}^{2}\right)\right] \leq\left(1-128 \alpha(2 T)^{2(H-\beta)}\right)^{-1 / 2} .
$$

Moreover, we have the following moment estimate for any $k \geq 1$ :

$$
\mathbb{E}\left(\left\|B^{H}\right\|_{0, T, \beta}^{2 k}\right) \leq 32^{k}(2 T)^{2 p(H-\beta)} \frac{(2 k) !}{k !} .
$$

For any $\alpha \in(0,1)$, let $C^{\alpha}(a, b)$ be the space of $\alpha$-Hölder continuous functions $f$ on the interval $[a, b]$ and set

$$
\|f\|_{a, b, \alpha}:=\sup _{a \leq s \leq t \leq b} \frac{|f(t)-f(s)|}{|t-s|^{\alpha}} .
$$

Besides, for any continuous function $f \in C\left([a, b] ; \mathbb{R}^{d}\right)$, let

$$
\|f\|_{a, b, \infty}=\sup _{a \leq s \leq b}|f(s)| \text {. }
$$

When $a=0, b=T$, we will simply write $\|f\|_{\alpha},\|f\|_{\infty}$ for $\|f\|_{0, T, \alpha},\|f\|_{0, T, \infty}$, respectively.

\section{$3 \quad$ Main results}

Let $\left(\mathbb{R}^{d},\langle\cdot, \cdot\rangle,|\cdot|\right)$ be the $d$-dimensional Euclidean space with the inner product $\langle\cdot, \cdot\rangle$, which induces the norm $|\cdot|$. Let $\mathbb{R}^{d} \otimes \mathbb{R}^{m}$ be the set of all $d \times m$-matrices. Let $\tau>0$ be a fixed number and $\mathscr{C}=C\left([-\tau, 0] ; \mathbb{R}^{d}\right)$, which is endowed with the uniform norm $\|f\|_{\infty}:=$ $\sup _{-\tau \leq \theta \leq 0}|f(\theta)|$. For $f \in C\left([-\tau, \infty) ; \mathbb{R}^{d}\right)$ and fixed $t>0$, define the segment $f_{t} \in \mathscr{C}$ by $f_{t}(\theta)=f(t+\theta), \theta \in[-\tau, 0]$. For $a \geq 0,[a]$ stipilates the integer part of $a$. Let $\mathscr{B}_{b}\left(\mathbb{R}^{d}\right)$ be the collection of all bounded measurable functions on $\mathbb{R}^{d}$. 
In this paper, for $H \in\left(\frac{1}{2}, 1\right)$, we consider the following equation:

$$
\mathrm{d} X(t)=\left\{b(X(t))+\sigma Z\left(X_{t}\right)\right\} \mathrm{d} t+\sigma \mathrm{d} B^{H}(t), t>0
$$

with the initial datum $X_{0}=\xi \in \mathscr{C}$, where $\sigma \in \mathbb{R}^{d} \otimes \mathbb{R}^{m}, b: \mathbb{R}^{d} \rightarrow \mathbb{R}^{d}, d \geq m$ and $Z$ : $\mathscr{C} \rightarrow \mathbb{R}^{m}$ are measurable, $X_{t}$ is the segment process of $X(t)$ defined by $X_{t}(\theta)=X(t+\theta), \theta \in$ $[-\tau, 0], B^{H}(t)$ is an $m$-dimensional $\mathrm{fBM}$ on the complete probability space $\left(\Omega, \mathscr{F}_{,}\left(\mathscr{F}_{t}\right)_{t \geq 0}, \mathbb{P}\right)$. Consider a reference SDE as follows:

$$
\mathrm{d} Y(t)=b(Y(t)) \mathrm{d} t+\sigma \mathrm{d} B^{H}(t), \quad t>0, Y(0) \in \mathbb{R}^{d} .
$$

Let $\xi \in \mathscr{C}$, and let $Y^{\xi(0)}(\cdot)$ be a solution of $(3.2)$ with $Y^{\xi(0)}(0)=\xi(0)$. We extend $Y^{\xi(0)}(\cdot)$ from $[0, \infty)$ to $[-\tau, \infty)$ in the following way:

$$
Y^{\xi}(t)=\xi(t) I_{[-\tau, 0)}(t)+Y^{\xi(0)}(t) I_{[0, \infty)}(t), t \in[-\tau, \infty), \xi \in \mathscr{C}
$$

Then the weak existence and uniqueness of solutions to (3.1) and the weak convergence of EM scheme will be studied by using Girsanov's transform and the extended solutions to the reference equation (3.2).

We first introduce the following assumptions on $b$ and $Z$ for the weak existence and uniqueness result.

(A1) There exists a constant $K_{1} \in \mathbb{R}$ such that

$$
\langle b(x)-b(y), x-y\rangle \leq K_{1}|x-y|^{2}, \quad x, y \in \mathbb{R}^{d} .
$$

(A2) There exist $C_{1}>0$ and $q_{0} \geq 0$ such that $|b(x)| \leq C_{1}\left(1+|x|^{q_{0}}\right), \quad x \in \mathbb{R}^{d}$.

(A3) There exist $\alpha \in(H-1 / 2,1], p>0, C_{2}>0, C_{3} \geq 0$ and $q_{1} \geq 0$ such that

$$
\begin{aligned}
\left|Z\left(\eta_{1}\right)-Z\left(\eta_{2}\right)\right| & \leq C_{2}\left\|\eta_{1}-\eta_{2}\right\|_{\infty}^{\alpha}\left(1+\left\|\eta_{1}\right\|_{\infty}^{p}+\left\|\eta_{2}\right\|_{\infty}^{p}\right), \\
\left\langle\sigma Z\left(\eta_{1}+\eta_{2}\right), \eta_{1}(0)\right\rangle & \leq C_{3}\left(1+\left\|\eta_{2}\right\|_{\infty}^{q_{1}}+\left\|\eta_{1}\right\|_{\infty}^{2}\right), \eta_{1}, \eta_{2} \in \mathscr{C} .
\end{aligned}
$$

Our result on existence and uniqueness of weak solutions to (3.1) is the following theorem.

Theorem 3.1. Assume (A1)-(A3). For any $\xi \in \mathscr{C}$ with $\theta \in\left(\frac{2 H-1}{2 \alpha}, 1\right]$ and $\bar{C}_{1}>0$ such that

$$
|\xi(r)-\xi(s)| \leq \bar{C}_{1}|r-s|^{\theta}, \quad-\tau \leq r \leq s \leq 0
$$

then the equation (3.1) has a unique weak solution with $X_{0}=\xi$.

Remark 3.1. The condition (3.6) is for us to use Girsanov's transformation to remove the drift term $Z(\cdot)$ of equation (3.1). Given $T>0$. For any $\gamma \in C\left([-\tau, T], \mathbb{R}^{d}\right)$ with $\gamma_{0}=\xi_{0}$, to ensure that $\left\{\int_{0}^{s} Z\left(\gamma_{r}\right) \mathrm{d} r\right\}_{s \in[0, T]}$ belongs to the Cameron-Martin space of the fBM, 
it is necessary that the integral $\int_{0}^{\cdot} Z\left(\gamma_{s}\right) \mathrm{d} s \in I_{0+}^{H+\frac{1}{2}}\left(L^{2}\left([0, T], \mathbb{R}^{d}\right)\right)$. This means we need $Z(\gamma.) \in I_{0+}^{H-\frac{1}{2}}\left(L^{2}\left([0, T], \mathbb{R}^{d}\right)\right)$. Note that for $t \in[0, T \wedge \tau]$, we have

$$
\|\gamma \cdot\|_{0, t, \alpha}=\sup _{0 \leq r \leq u \leq t} \frac{\left\|\gamma_{u}-\gamma_{r}\right\|_{\infty}}{|u-r|^{\alpha}}=\sup _{0 \leq r \leq u \leq t, v \in[-\tau, 0]} \frac{|\gamma(u+v)-\gamma(r+v)|}{(u-r)^{\alpha}} \geq\|\xi\|_{-\tau, 0, \alpha} .
$$

Hence, despite imposing regularity conditions on $Z$, we also need an additional assumption on the initial value $\xi$. If $Z$ is $\alpha$-Hölder continuous and $\xi$ is $\theta$-Hölder continuous, then our conditions on $\xi$ yields that $\theta \alpha>H-\frac{1}{2}$, which ensure that $\left\{\int_{0}^{s} Z\left(\gamma_{r}\right) \mathrm{d} r\right\}_{s \in[0, T]}$ is in the Cameron-Martin space.

Next, we shall study the weak convergence of the numerical approximation to (3.1). In (3.1), $\sigma$ is a $d \times m$ matrix with $d \geq m$. For $d>m$, this equation is obviously degenerate. Hence, we shall introduce the pseudo-inverse of $\sigma$ to cover some degenerate models, such as stochastic Hamilton systems. Denote by $\operatorname{Ran}(\sigma)$ the range of $\sigma$, i.e. $\operatorname{Ran}(\sigma)=\sigma\left(\mathbb{R}^{m}\right)$. If $\operatorname{Ran}(\sigma)$ contains nonzero vectors, then $\sigma \sigma^{*}$ is a bijective from $\operatorname{Ran}(\sigma)$ onto $\operatorname{Ran}(\sigma)$, whose inverse is denoted by $\left.\left(\sigma \sigma^{*}\right)^{-1}\right|_{\operatorname{Ran}(\sigma)}$. Let $\pi_{*}$ be the orthogonal projection from $\mathbb{R}^{d}$ to $\operatorname{Ran}(\sigma)$. Then $\mathbb{R}^{d}$ has the following decomposition:

$$
\mathbb{R}^{d}=\pi_{*} \mathbb{R}^{d} \oplus\left(I_{d \times d}-\pi_{*}\right) \mathbb{R}^{d} \equiv \operatorname{Ran}(\sigma) \oplus\left(I_{d \times d}-\pi_{*}\right) \mathbb{R}^{d},
$$

where $I_{d \times d}$ is the identity matrix of $\mathbb{R}^{d}$. We define $\widehat{\sigma}^{-1}$, the pseudo-inverse of $\sigma$, as follows

$$
\widehat{\sigma}^{-1} v=\sigma^{*}\left(\left.\left(\sigma \sigma^{*}\right)^{-1}\right|_{\operatorname{Ran}(\sigma)} \pi_{*} v\right), v \in \mathbb{R}^{d} .
$$

Then $\left\|\widehat{\sigma}^{-1}\right\|=\left\|\left.\left(\sigma \sigma^{*}\right)^{-1}\right|_{\operatorname{Ran}(\sigma)}\right\|$. In particular, if $\sigma$ is of the form $\left(\begin{array}{c}0 \\ \sigma_{0}\end{array}\right)$ with $\sigma_{0}$ is an invertible $m \times m$-matrix and 0 is a $(d-m) \times m$ zero matrix, then

$$
\widehat{\sigma}^{-1}=\left(0^{*}, \sigma_{0}^{-1}\right), \quad\left\|\widehat{\sigma}^{-1}\right\|=\left\|\sigma_{0}^{-1}\right\| .
$$

We need stronger assumptions on $b$ and $Z$ for numerical approximation.

(H1) (A1) holds and there exists a constant $L_{1}>0$ such that

$$
|b(x)-b(y)| \leq L_{1}|x-y|, x, y \in \mathbb{R}^{d} .
$$

Moreover, if $\operatorname{Ran}(\sigma) \neq \mathbb{R}^{d}$, we also assume that there exist a matrix $A$ on $\left(I_{d \times d}-\pi_{*}\right)\left(\mathbb{R}^{d}\right)$ and a measurable function $b_{*}: \operatorname{Ran}(\sigma) \rightarrow\left(I_{d \times d}-\pi_{*}\right)\left(\mathbb{R}^{d}\right)$ such that

$$
\left(I_{d \times d}-\pi_{*}\right) b(x)=A\left(I_{d \times d}-\pi_{*}\right) x+b_{*}\left(\pi_{*} x\right), x \in \mathbb{R}^{d} .
$$

(H2) $Z$ is Hölder continuous with the exponent $\alpha \in\left(1-\frac{1}{2 H}, 1\right]$, that is

$$
|Z(\xi)-Z(\eta)| \leq L_{2}\|\xi-\eta\|_{\infty}^{\alpha}, \xi, \eta \in \mathscr{C}
$$


(H3) the initial value $\xi \in \mathscr{C}$ is Hölder continuous with exponent $\theta \in\left(\frac{2 H-1}{2 \alpha}, 1\right]$, that is,

$$
|\xi(t)-\xi(s)| \leq L_{3}|t-s|^{\theta}, \quad s, t \in[-\tau, 0]
$$

By these conditions, it follows from Theorem 3.1 that (3.1) has a unique weak solution with $X_{0}=\xi$.

Remark 3.2. Since the pseudo-inverse of $\sigma$ is the inverse of $\sigma$ if it is invertible, our setting can unify non-degenerate and some degenerate models. A typical example for the equation with $\{0\} \subsetneq \operatorname{Ran}(\sigma) \subsetneq \mathbb{R}^{d}$ is the following stochastic Hamiltonian system $(d=2 m)$ :

$$
\left\{\begin{array}{l}
\mathrm{d} X^{(1)}(t)=X^{(2)}(t) \mathrm{d} t \\
\mathrm{~d} X^{(2)}(t)=b_{0}\left(X^{(1)}(t), X^{(2)}(t)\right) \mathrm{d} t+Z_{0}\left(X_{t}^{(1)}, X_{t}^{(2)}\right) \mathrm{d} t+\sigma_{0} \mathrm{~d} B^{H}(t)
\end{array}\right.
$$

where $\sigma_{0}$ is an invertible $m \times m$-matrix. For any $\eta_{1}, \eta_{2} \in \mathscr{C}, x=\left(x^{(1)}, x^{(2)}\right) \in \mathbb{R}^{2 m}$, we set

$$
b\left(x^{(1)}, x^{(2)}\right)=\left(\begin{array}{c}
x^{(2)} \\
b_{0}\left(x^{(1)}, x^{(2)}\right)
\end{array}\right), \quad Z\left(\eta_{1}, \eta_{2}\right)=\left(\begin{array}{c}
0 \\
\sigma_{0}^{-1} Z_{0}\left(\eta_{1}, \eta_{2}\right)
\end{array}\right), \quad \sigma=\left(\begin{array}{c}
0 \\
\sigma_{0}
\end{array}\right),
$$

Then

$$
\mathrm{d} X(t) \equiv \mathrm{d}\left(\begin{array}{c}
X^{(1)}(t) \\
X^{(2)}(t)
\end{array}\right)=\left(b(X(t))+\sigma Z\left(X_{t}\right)\right) \mathrm{d} t+\sigma \mathrm{d} B^{H}(t)
$$

and in this case, $\pi_{*}\left(x^{(1)}, x^{(2)}\right)=\left(0, x^{(2)}\right), b_{*}\left(\left(0, x^{(2)}\right)\right)=\left(x^{(2)}, 0\right)$ and $A \equiv 0$ in $(\mathrm{A} 1)$.

We can construct the EM scheme now. Let $\delta \in(0,1)$ be the step-size given by $\delta=\tau / M$ for some $M \in \mathbb{N}$ sufficiently large. The continuous time EM scheme associated with (3.1) is defined as below:

$$
\mathrm{d} X^{(\delta)}(t)=\left\{\left(I_{d \times d}-\pi_{*}\right) b\left(X^{(\delta)}(t)\right)+\pi_{*} b\left(X^{(\delta)}\left(t_{\delta}\right)\right)+\sigma Z\left(\widehat{X}_{t}^{(\delta)}\right)\right\} \mathrm{d} t+\sigma \mathrm{d} B^{H}(t), \quad t>0,
$$

with the initial value $X^{(\delta)}(u)=X(u)=\xi(u), u \in[-\tau, 0]$, where $t_{\delta}:=[t / \delta] \delta$ and $\widehat{X}_{t}^{(\delta)} \in \mathscr{C}$ defined as follows

$$
\widehat{X}_{t}^{(\delta)}(u)=X^{(\delta)}\left((t+u) \wedge t_{\delta}\right), u \in[-\tau, 0] .
$$

For $t \in[0, \delta), \widehat{X}_{t}^{(\delta)}(u)=X^{(\delta)}((t+u) \wedge 0)=\xi((t+u) \wedge 0)$, and

$$
H(t):=\pi_{*} X^{(\delta)}(t)=\pi_{*} X^{(\delta)}(0)+\pi_{*} b\left(X^{(\delta)}(0)\right) t+\int_{0}^{t} \sigma Z\left(\widehat{X}_{s}^{(\delta)}\right) \mathrm{d} s+\sigma B^{H}(t) .
$$

Then it follows from (H1) that

$$
\left(I_{d \times d}-\pi_{*}\right) X^{(\delta)}(t)=\left(I_{d \times d}-\pi_{*}\right) X^{(\delta)}(0)+\int_{0}^{t}\left(I_{d \times d}-\pi_{*}\right) b\left(\pi_{*} X^{(\delta)}(s)+\left(I_{d \times d}-\pi_{*}\right) X^{(\delta)}(s)\right) \mathrm{d} s
$$




$$
=\left(I_{d \times d}-\pi_{*}\right) X^{(\delta)}(0)+\int_{0}^{t} A\left(I_{d \times d}-\pi_{*}\right) X^{(\delta)}(s) \mathrm{d} s+\int_{0}^{t} b_{*}(H(s)) \mathrm{d} s,
$$

which implies that

$$
\left(I_{d \times d}-\pi_{*}\right) X^{(\delta)}(t)=\mathrm{e}^{A t}\left(I_{d \times d}-\pi_{*}\right) X^{(\delta)}(0)+\int_{0}^{t} \mathrm{e}^{A(t-s)} b_{*}(H(s)) \mathrm{d} s .
$$

Thus, $X^{(\delta)}(t)=\left(I_{d \times d}-\pi_{*}\right) X^{(\delta)}(t)+\pi_{*} X^{(\delta)}(t)$ can be obtained explicitly on $[0, \delta]$. By induction, we can get $X^{(\delta)}(t)$ explicitly.

Let

$$
\bar{K}_{1}=2 K_{1}+\mathbb{1}_{\left[K_{1} \geq 0\right]}+\frac{\left|K_{1}\right|}{2} \mathbb{1}_{\left[K_{1}<0\right]}, \quad \bar{K}_{2}=\mathbb{1}_{\left[K_{1} \geq 0\right]}+\frac{2}{\left|K_{1}\right|} \mathbb{1}_{\left[K_{1}<0\right]},
$$

and

$$
\Phi\left(\bar{K}_{1}, \bar{K}_{2}, T\right)=\sqrt{\frac{\bar{K}_{2}\left(\mathrm{e}^{\bar{K}_{1} T}-1\right)}{\bar{K}_{1}}} .
$$

Our main result on the weak convergence of EM scheme to (3.1) is stated as follows.

Theorem 3.2. Assume (H1)-(H3) and $\operatorname{Ran}(\sigma) \neq\{0\}$. For $\delta \in(0,1)$ if $T$ satisfies

$$
\left\{\frac{12 L_{2}^{2} T^{2-2 H}\left(1+(H-1 / 2) C_{0}\right)^{2}}{(1-H) \Gamma^{2}(3 / 2-H)}\|\sigma\|^{2}\right\} \mathbb{1}_{[\alpha=1]}<\left(L_{1} \Phi\left(\bar{K}_{1}, \bar{K}_{2}, T\right)+1\right)^{-2} \frac{1}{2 T},
$$

and

$$
\begin{aligned}
& \frac{6 L_{1}^{2}\|\sigma\|^{2}\left\|\widehat{\sigma}^{-1}\right\|^{2}}{\Gamma^{2}\left(\frac{3}{2}-H\right)(1-H)}\left(1+L_{1} T\left(L_{1} \Phi\left(\bar{K}_{1}, \bar{K}_{2}, T\right)+1\right)\right)^{2}\left\{3\left[1+C_{0}\left(H-\frac{1}{2}\right)\right]^{2} T^{2-2 H} \delta^{2 \beta}\right. \\
& \quad+2(2 H-1)^{2} T \delta^{2 \beta+1-2 H}\left[\frac{1}{(1+2 \beta-2 H)^{2}}+\frac{2^{4 H-1}}{(3-2 H)^{2}}+\frac{2^{2 H-1}}{(2 H-1)^{2}(1-H)}\right. \\
& \left.\left.\quad+\frac{\mathcal{B}^{2}\left(\frac{3}{2}-H, \beta+\frac{1}{2}-H\right)(1-H) \delta}{8(1+\beta-H) T}\right]\right\} \\
& \quad+\frac{6 L_{2}^{2} T^{2 H-1}(2 H-1)^{2}}{\Gamma^{2}\left(\frac{3}{2}-H\right)}\left(1+L_{1} T\left(L_{1} \Phi\left(\bar{K}_{1}, \bar{K}_{2}, T\right)+1\right)\right)^{2 \alpha}\|\sigma\|^{2 \alpha} \\
& \quad\left\{\frac{\mathcal{B}^{2}\left(\frac{3}{2}-H, \alpha(\beta \wedge \theta)+1 / 2-H\right) T^{2 \alpha(\beta \wedge \theta)+3-4 H}}{2 \alpha(\beta \wedge \theta)+3-4 H}\right. \\
& \left.\quad+\frac{\delta^{2 \alpha(\beta \wedge \theta)+1-2 H} T^{2-2 H}}{1-H}\left(\frac{16^{H}}{(3-2 H)^{2}}+\frac{2^{2 H}}{(2 H-1)^{2}(1-H)}\right)\right\} \mathbb{1}_{[\alpha=1]}<1 /\left(128(2 T)^{2(H-\beta)}\right),
\end{aligned}
$$

where $\beta \in\left(\frac{2 H-1}{2 \alpha}, H\right)$ and $C_{0}=\int_{0}^{1} \frac{u^{\frac{1}{2}-H}-1}{(1-u)^{\frac{1}{2}+H}} \mathrm{~d} u$, then for any bounded measurable function $f$ on $\mathbb{R}^{d}$, there exists a constant $C_{T}$ such that for $t \in[0, T]$

$$
\left|\mathbb{E} f(X(t))-\mathbb{E} f\left(X^{(\delta)}(t)\right)\right| \leq C_{T} \delta^{\alpha(\beta \wedge \theta)+\frac{1}{2}-H} .
$$

Remark 3.3. The convergence result only holds for $t \in[0, T]$ and $T$ satisfies (3.11) and (3.12). It is not difficult to see that for any fix $\delta \in(0,1)$, there always exists $T>0$ such that (3.11) and (3.12) hold, and $T$ is a decreasing function of $\delta$. 


\section{Proof of Theorem 3.1}

We first introduce the following lemma on the existence and uniqueness of solutions to (3.2).

Lemma 4.1. Assume (A1). Then (3.2) has a unique strong solution and

$$
|Y(t)| \leq \mathrm{e}^{\frac{\bar{K}_{2} t}{2}}|Y(0)|+\sqrt{\bar{K}_{2}}\left(\int_{0}^{t} e^{\bar{K}_{1}(t-r)}\left|b\left(\sigma B^{H}(r)\right)\right|^{2} \mathrm{~d} r\right)^{\frac{1}{2}}+\left|\sigma B^{H}(t)\right|, t \geq 0 .
$$

Furthermore, if (A2) holds, then

$$
\mathbb{E}\|Y\|_{0, t, \beta}^{q}<\infty, q>0, t>0,0<\beta<H
$$

Proof. (1) Let $U(t)=Y(t)-\sigma B^{H}(t)$. Then $U(t)$ satisfies

$$
\mathrm{d} U(t)=b\left(U(t)+\sigma B^{H}(t)\right) \mathrm{d} t, U(0)=Y(0) .
$$

Set $\bar{b}(u, t)=b\left(u+\sigma B^{H}(t)\right)$. Then it is easy to see that

$$
\left\langle\bar{b}\left(u_{1}, t\right)-\bar{b}\left(u_{2}, t\right), u_{1}-u_{2}\right\rangle \leq K_{1}\left|u_{1}-u_{2}\right|^{2}
$$

which implies that (4.2) has a unique solution. Moreover, it follows from the chain rule and the Hölder inequality that

$$
\begin{aligned}
\mathrm{d}|U(t)|^{2} & =2\langle\bar{b}(U(t), t), U(t)\rangle \mathrm{d} t \\
& \leq 2 K_{1}|U(t)|^{2}+2\left\langle b\left(\sigma B^{H}(t)\right), U(t)\right\rangle \mathrm{d} t \\
& \leq \bar{K}_{1}|U(t)|^{2} \mathrm{~d} t+\bar{K}_{2}\left|b\left(\sigma B^{H}(t)\right)\right|^{2} \mathrm{~d} t
\end{aligned}
$$

Then for any $t \geq s$

$$
\begin{gathered}
|Y(t)| \leq \mathrm{e}^{\frac{1}{2}(t-s) \bar{K}_{1}}|Y(s)|+\sqrt{\bar{K}_{2}}\left(\int_{s}^{t} e^{\bar{K}_{1}(t-r)}\left|b\left(\sigma B^{H}(r)\right)\right|^{2} \mathrm{~d} r\right)^{\frac{1}{2}} \\
+\mathrm{e}^{\frac{\bar{K}_{1}(t-s)}{2}}\left|\sigma B^{H}(s)\right|+\left|\sigma B^{H}(t)\right|,
\end{gathered}
$$

which implies our first claim.

(2) For any $0<\beta<H$,

$$
\begin{aligned}
\frac{|Y(t)-Y(s)|}{(t-s)^{\beta}} \leq & \frac{1}{(t-s)^{\beta}} \int_{s}^{t}|b(Y(r))| \mathrm{d} r+\|\sigma\|\left\|B^{H}\right\|_{0, t, \beta} \\
\leq & \frac{C_{1}}{(t-s)^{\beta}} \int_{s}^{t}\left(1+|Y(r)|^{q_{0}}\right) \mathrm{d} r+\|\sigma\|\left\|B^{H}\right\|_{0, t, \beta} \\
\leq & C_{1}(t-s)^{1-\beta}+\|\sigma\|\left\|B^{H}\right\|_{0, t, \beta}+C_{1} 3^{\left(q_{0}-1\right)^{+}}(t-s)^{1-\beta} \mathrm{e}^{\frac{t \bar{K}_{1}^{+} q_{0}}{2}}|Y(0)|^{q_{0}} \\
& \quad+C_{1} 3^{\left(q_{0}-1\right)^{+}}\left(\sqrt{\bar{K}_{2}} C_{1}\left(1+\|\sigma\|^{q_{0}}\left\|B^{H}\right\|_{0, t, \infty}^{q_{0}}\right)\right)^{q_{0}} \mathrm{e}^{\frac{q_{0} \bar{K}_{1}^{+} t}{2}} t^{q_{0}}(t-s)^{1-\beta}
\end{aligned}
$$




$$
+C_{1} 3^{\left(q_{0}-1\right)^{+}}\left(\|\sigma\|\left\|B^{H}\right\|_{0, t, \infty}\right)^{q_{0}}(t-s)^{1-\beta}
$$

which yields

$$
\begin{aligned}
& \|Y\|_{0, t, \beta} \leq C_{1} t^{1-\beta}+\|\sigma\|\left\|B^{H}\right\|_{0, t, \beta}+C_{1} 3^{\left(q_{0}-1\right)^{+}} t^{1-\beta}\|\sigma\|^{q_{0}}\left\|B^{H}\right\|_{0, t, \infty}^{q_{0}} \\
& \quad+C_{1} 3^{\left(q_{0}-1\right)^{+}} t^{1-\beta} \mathrm{e}^{\frac{t \bar{K}_{1}^{+} q_{0}}{2}}\left(\|Y\|_{0, t, \infty}+t^{q_{0}}\left(\sqrt{\bar{K}_{2}} C_{1}\left(1+\|\sigma\|^{q_{0}}\left\|B^{H}\right\|_{0, t, \infty}^{q_{0}}\right)\right)^{q_{0}}\right) .
\end{aligned}
$$

Combining this with (4.1), it is clear that our second claim holds.

Next lemma is to investigate the exponential martingale, which is crucial to prove Theorem 3.1. Fix any $T>0$. Let

$$
\begin{aligned}
&\left\{\widetilde{B}^{H}(t)\right\}_{t \in[0, T]}=\left\{B^{H}(t)-\int_{0}^{t} Z\left(Y_{s}^{\xi}\right) \mathrm{d} s\right\}_{t \in[0, T]}, \\
& R^{\xi}(t)=\exp \left(\int_{0}^{t}\left\langle K_{H}^{-1}\left(\int_{0}^{\cdot} Z\left(Y_{r}^{\xi}\right) \mathrm{d} r\right)(s), \mathrm{d} B(s)\right\rangle\right. \\
&\left.-\frac{1}{2} \int_{0}^{t}\left|K_{H}^{-1}\left(\int_{0}^{\cdot} Z\left(Y_{r}^{\xi}\right) \mathrm{d} r\right)\right|^{2}(s) \mathrm{d} s\right), t \in[0, T] .
\end{aligned}
$$

Lemma 4.2. Let the assumptions of Theorem 3.1 hold. Then

(1) $\left\{\widetilde{B}^{H}(t)\right\}_{t \in[0, T]}$ is a fractional Brownian motion under $R^{\xi}(T) \mathbb{P}$.

(2) Assume in addition that $q_{0}=1$ in (A2). If there exist $C_{4} \geq 0, C_{5} \geq 0$ and $p \in(0,1)$ such that

$$
\left|Z\left(\eta_{1}\right)-Z\left(\eta_{2}\right)\right| \leq C_{4}\left\{\left\|\eta_{1}-\eta_{2}\right\|_{\infty}^{\alpha} \wedge\left(1+C_{5}\left(\left\|\eta_{1}\right\|_{\infty}^{p}+\left\|\eta_{2}\right\|_{\infty}^{p}\right)\right)\right\}
$$

then for any $C \geq 0$

$$
\mathbb{E} \exp \left\{C \int_{0}^{T}\left|K_{H}^{-1}\left(\int_{0} Z\left(Y_{r}^{\xi}\right) \mathrm{d} r\right)\right|^{2}(s) \mathrm{d} s\right\}<\infty .
$$

(3) If (4.5) holds with $p=1$ and $T>0$ is small enough such that

$$
\begin{aligned}
& \left(\frac{3 C_{4}^{2} T^{2-2 H}\left(1+(H-1 / 2)^{2} C_{0}^{2}\right)}{(1-H) \Gamma^{2}(3 / 2-H)} \mathbf{1}_{[\alpha=1]}+\frac{3 C_{6} C_{5}^{2} T^{2-2 T}(H-1 / 2)^{2}}{(1-H) \Gamma^{2}(3 / 2-H)}\right)\|\sigma\|^{2} \\
& <\left(L_{1} \Phi\left(\bar{K}_{1}, \bar{K}_{2}, T\right)+1\right)^{-2} \frac{1}{2 T}
\end{aligned}
$$

where $C_{0}$ is defined in Theorem 3.2, then (4.6) holds for some $C>1$. 
Proof. If (3.6) holds for $\theta \geq H$, then (3.6) holds for $\theta \in(H-1 / 2, H)$. Hence, we shall assume that $\theta \in(H-1 / 2, H)$ in the following proof.

(1) It follows from (2.3) that

$$
\begin{aligned}
K_{H}^{-1}\left(\int_{0} Z\left(Y_{r}^{\xi}\right) \mathrm{d} r\right)(s)= & s^{H-\frac{1}{2}} D_{0+}^{H-\frac{1}{2}}\left(\cdot^{\frac{1}{2}-H} Z\left(Y_{\cdot}^{\xi}\right)\right)(s) \\
= & \frac{H-\frac{1}{2}}{\Gamma\left(\frac{3}{2}-H\right)}\left[\frac{s^{\frac{1}{2}-H}}{H-\frac{1}{2}} Z\left(Y_{s}^{\xi}\right)+s^{H-\frac{1}{2}} Z\left(Y_{s}^{\xi}\right) \int_{0}^{s} \frac{s^{\frac{1}{2}-H}-r^{\frac{1}{2}-H}}{(s-r)^{\frac{1}{2}+H}} \mathrm{~d} r\right. \\
& \left.\quad+s^{H-\frac{1}{2}} \int_{0}^{s} \frac{Z\left(Y_{s}^{\xi}\right)-Z\left(Y_{r}^{\xi}\right)}{(s-r)^{\frac{1}{2}+H}} r^{\frac{1}{2}-H} \mathrm{~d} r\right] \\
= & \frac{H-\frac{1}{2}}{\Gamma\left(\frac{3}{2}-H\right)}\left(J_{1}(s)+J_{2}(s)+J_{3}(s)\right) .
\end{aligned}
$$

By (3.4), we have

$$
\begin{aligned}
& \left|J_{1}(s)\right|^{2}+\left|J_{2}(s)\right|^{2} \\
& \leq \frac{s^{1-2 H}}{\left(H-\frac{1}{2}\right)^{2}}\left(C_{2}\left(1+\left\|Y_{s}^{\xi}\right\|_{\infty}^{p}\right)\left\|Y_{s}^{\xi}\right\|_{\infty}^{\alpha}+|Z(0)|\right)^{2} \\
& +s^{2 H-1}\left|\int_{0}^{s} \frac{s^{\frac{1}{2}-H}-r^{\frac{1}{2}-H}}{(s-r)^{\frac{1}{2}+H}} \mathrm{~d} r\right|^{2}\left(C_{2}\left(1+\left\|Y_{s}^{\xi}\right\|_{\infty}^{p}\right)\left\|Y_{s}^{\xi}\right\|_{\infty}^{\alpha}+|Z(0)|\right)^{2} \\
& \leq 3 s^{1-2 H}\left(\frac{4}{(2 H-1)^{2}}+C_{0}^{2}\right)\left(|Z(0)|^{2}+C_{2}^{2}\left(\left\|Y_{s}^{\xi}\right\|_{\infty}^{2 \alpha}+\left\|Y_{s}^{\xi}\right\|_{\infty}^{2(p+\alpha)}\right)\right),
\end{aligned}
$$

and

$$
\begin{aligned}
\left|J_{3}(s)\right| & \leq C_{2} s^{H-\frac{1}{2}} \int_{0}^{s} \frac{\left\|Y_{s}^{\xi}-Y_{r}^{\xi}\right\|_{\infty}^{\alpha}\left(1+\left\|Y_{s}^{\xi}\right\|_{\infty}^{p}+\left\|Y_{r}^{\xi}\right\|_{\infty}^{p}\right)}{(s-r)^{H+1 / 2} r^{H-1 / 2}} \mathrm{~d} r \\
& \leq 2 C_{2} s^{H-\frac{1}{2}}\left(1+\left\|Y^{\xi}\right\|_{-\tau, s, \infty}^{p}\right) \int_{0}^{s} \frac{\left\|Y^{\xi}\right\|_{-\tau, r, \theta}^{\alpha}(s-r)^{\theta \alpha}}{(s-r)^{H+1 / 2} r^{H-1 / 2}} \mathrm{~d} r \\
& =2 C_{2} s^{\theta \alpha+\frac{1}{2}-H} \mathcal{B}\left(\frac{3}{2}-H, \theta \alpha+\frac{1}{2}-H\right)\left(1+\left\|Y^{\xi}\right\|_{-\tau, s, \infty}^{p}\right)\left\|Y^{\xi}\right\|_{-\tau, s, \theta}^{\alpha},
\end{aligned}
$$

where $\mathcal{B}$ is Beta function.

Combining this with (4.9), we arrive at

$$
\begin{aligned}
& \left|K_{H}^{-1}\left(\int_{0}^{\cdot} Z\left(Y_{r}^{\xi}\right) \mathrm{d} r\right)\right|^{2}(s) \\
& \leq 3 s^{1-2 H}\left(\frac{4}{(2 H-1)^{2}}+C_{0}^{2}\right)\left(|Z(0)|^{2}+C_{2}^{2}\left(\left\|Y_{s}^{\xi}\right\|_{\infty}^{2 \alpha}+\left\|Y_{s}^{\xi}\right\|_{\infty}^{2(p+\alpha)}\right)\right) \\
& +4 C_{2}^{2} s^{2 \theta \alpha+1-2 H} \mathcal{B}^{2}\left(\frac{3}{2}-H, \theta \alpha+\frac{1}{2}-H\right)\left(1+\left\|Y^{\xi}\right\|_{-\tau, s, \infty}^{2 p}\right)\left\|Y_{s}^{\xi}\right\|_{-\tau, s, \theta}^{2 \alpha},
\end{aligned}
$$


Let

$$
\tau_{n}=\inf \left\{t>\left.0\left|\int_{0}^{t}\right| K_{H}^{-1}\left(\int_{0} Z\left(Y_{r}^{\xi}\right) \mathrm{d} r\right)\right|^{2}(s) \mathrm{d} s \geq n\right\}, \quad n \in \mathbb{N} .
$$

Thus, we know $R^{\xi}\left(T \wedge \tau_{n}\right)$ which was defined in (4.4) is an exponential martingale for $t \in\left[0, T \wedge \tau_{n}\right]$. The Girsanov theorem ([23, Proposition 4.1.2]) implies that

$$
\widetilde{B}_{n}(t):=B(t)-\int_{0}^{t \wedge \tau_{n}} K_{H}^{-1}\left(\int_{0} Z\left(Y_{r}^{\xi}\right) \mathrm{d} r\right)(s) \mathrm{d} s, t \geq 0
$$

is a Brownian motion under $R^{\xi}\left(T \wedge \tau_{n}\right) \mathbb{P}$. This implies

$$
\widetilde{B}_{n}^{H}(t):=B^{H}(t)-\int_{0}^{t \wedge \tau_{n}} Z\left(Y_{r}^{\xi}\right) \mathrm{d} r, t \geq 0
$$

is a $\mathrm{fBM}$ under $R^{\xi}\left(T \wedge \tau_{n}\right) \mathbb{P}$ and $Y^{\xi}$ satisfies

$$
\mathrm{d} Y^{\xi}(t)=b\left(Y^{\xi}(t)\right) \mathrm{d} t+\sigma \mathrm{d} \widetilde{B}_{n}^{H}(t)+\mathbf{1}_{\left[0 \leq t \leq \tau_{n}\right]} \sigma Z\left(Y_{t}^{\xi}\right) \mathrm{d} t .
$$

Let $u^{\xi}(t)=Y^{\xi}(t)-\sigma \widetilde{B}_{n}^{H}(t)$, Then for $0 \leq t \leq \tau_{n}$, we derive from (3.5)

$$
\begin{aligned}
\mathrm{d} u^{\xi}(t)^{2} & =2\left\langle b\left(u^{\xi}(t)+\sigma \widetilde{B}_{n}^{H}(t), u^{\xi}(t)\right)\right\rangle \mathrm{d} t+2\left\langle\sigma Z\left(u_{t}^{\xi}+\sigma \widetilde{B}_{n}^{H}(t)\right), u^{\xi}(t)\right\rangle \mathrm{d} t \\
& \leq \bar{K}_{1}\left(u^{\xi}(t)^{2}\right) \mathrm{d} t+C_{1}^{2} \bar{K}_{2}\left(1+\left|\sigma \widetilde{B}_{n}^{H}(t)\right|^{q_{0}}\right)^{2} \mathrm{~d} t \\
& +2 C_{3}\left(1+\left\|u_{t}^{\xi}\right\|_{\infty}^{2}+\|\sigma\|^{q_{1}}\left\|\widetilde{B}_{n, t}^{H}\right\|_{\infty}^{q_{1}}\right) \mathrm{d} t .
\end{aligned}
$$

Then, we know

$$
\begin{aligned}
\left|Y^{\xi}(t)\right|^{2} & \leq\left|Y^{\xi}(0)\right|^{2}+2\left(\bar{K}_{1}+2 C_{3}\right) \int_{0}^{t}\left\|Y_{s}^{\xi}\right\|_{\infty}^{2} \mathrm{~d} s+2 \bar{K}_{2} C_{1}^{2} \int_{0}^{t}\left(1+\left|\sigma \widetilde{B}_{n}^{H}(s)\right|^{q_{0}}\right)^{2} \mathrm{~d} s \\
& +4 C_{3} \int_{0}^{t}\left(1+\|\sigma\|^{q_{1}}\left\|\widetilde{B}_{n, s}^{H}\right\|_{\infty}^{q_{1}}\right) \mathrm{d} s+2\left|\sigma \widetilde{B}_{n}^{H}(t)\right|^{2} \\
& =: F^{2}\left(\widetilde{B}_{n}^{H}\right)(t)+2\left(\bar{K}_{1}+2 C_{3}\right) \int_{0}^{t}\left\|Y_{s}^{\xi}\right\|_{\infty}^{2} \mathrm{~d} s .
\end{aligned}
$$

Note that $\sup _{0 \leq s \leq t}\left\|Y_{s}^{\xi}\right\|_{\infty} \leq\|\xi\|_{\infty} \vee \sup _{0 \leq s \leq t}\left|Y^{\xi}(s)\right|$, we arrive at that for any $p \geq 2$

$$
\left.\left\|Y^{\xi}\right\|_{0, t, \infty}^{p} \leq 3^{p-1}\left(\|\xi\|_{\infty}^{p}\right)+F^{p}\left(\widetilde{B}_{n}^{H}\right)(t)+2^{p}\left(\bar{K}_{1}+2 C_{3}\right)^{p} t^{p-1} \int_{0}^{t}\left\|Y^{\xi}\right\|_{0, s, \infty}^{2} \mathrm{~d} s\right),
$$

Combining this with Gronwall's lemma, it yields that

$$
\mathbb{E}\left\|Y^{\xi}\right\|_{0, t, \infty}^{p} \leq 3^{p-1}\left(\|\xi\|_{\infty}^{p}+\mathbb{E} F^{p}\left(\widetilde{B}_{n}^{H}\right)(t)\right) \mathrm{e}^{(3 t)^{p-1} 2^{p}\left(\bar{K}_{1}+2 C_{3}\right)^{p}}<\infty,
$$

which, together with the Hölder inequality, yields that for any $p>0$,

$$
\mathbb{E}\left\|Y^{\xi}\right\|_{0, t, \infty}^{p}<\infty .
$$


Similarly, following the proof of (4.3), we get

$$
\begin{gathered}
\|Y\|_{0, t, \beta} \leq\left\{C_{1}\left(1+\left\|Y^{\xi}\right\|_{0, t, \infty}^{q_{0}}\right)+\|\sigma\||Z(0)|+C_{2}\|\sigma\|\left\|Y^{\xi}\right\|_{-\tau, t, \infty}^{\alpha}\left(1+\left\|Y^{\xi}\right\|_{-\tau, t, \infty}^{p}\right)\right\} t^{1-\beta} \\
+\|\sigma\|\left\|\widetilde{B}_{n}^{H}\right\|_{0, t, \beta}, t>0, \beta \in(0, H) .
\end{gathered}
$$

Combining this with (4.12), it yields that for any $p>0$

$$
\mathbb{E}\|Y\|_{0, t, \beta}^{p}<\infty .
$$

Combining this with (4.12) and (3.6), we obtain $\widetilde{B}_{n}^{H}$ under $R^{\xi}\left(T \wedge \tau_{n}\right) \mathbb{P}$ has the same distribution as $B^{H}$ under $\mathbb{P}$, we have

$$
\sup _{n} \mathbb{E} R^{\xi}\left(T \wedge \tau_{n}\right)\left(\left\|Y^{\xi}\right\|_{-\tau, T, \infty}^{q}+\left\|Y^{\xi}\right\|_{-\tau, T, \theta}^{q}\right)<\infty, \quad q>0, \theta<H .
$$

Then by (4.4), (4.11) and (4.12), we obtain

$$
\begin{array}{rl}
\sup _{t \in[0, T], n} & \mathbb{E} R^{\xi}\left(t \wedge \tau_{n}\right) \log R^{\xi}\left(t \wedge \tau_{n}\right) \\
\quad \leq C \sup _{t \in[0, T], n} \mathbb{E} R^{\xi}\left(t \wedge \tau_{n}\right)\left(1+\left\|Y^{\xi}\right\|_{-\tau, t, \infty}^{2(p+\alpha)}+\left\|Y^{\xi}\right\|_{-\tau, t, \theta}^{2 \alpha}+\left\|Y^{\xi}\right\|_{-\tau, t, \theta}^{2 \alpha}\|Y\|_{-\tau, t, \infty}^{2 p}\right) \\
\quad<\infty .
\end{array}
$$

Hence, it follows from the Fatou lemma and the martingale convergence theorem that $\left\{R^{\xi}(t)\right\}_{t \in[0, T]}$ is an uniformly integrable martingale and

$$
\sup _{t \in[0, T]} \mathbb{E} R^{\xi}(t) \log R^{\xi}(t)<\infty .
$$

It follows from Girsanov's theorem that under $R^{\xi}(T) \mathbb{P}$, the process $\widetilde{B}^{H}$ is a fBM.

(2) By (4.5), we have

$$
\begin{aligned}
& \left|J_{1}(s)\right|^{2}+\left|J_{2}(s)\right|^{2} \\
& \leq 2 C_{4}^{2} s^{1-2 H}\left(\frac{4}{(2 H-1)^{2}}+C_{0}^{2}\right)\left(\left(1+C_{5}\left\|Y^{\xi}\right\|_{-\tau, s, \infty}^{p}\right)^{2} \wedge\left\|Y^{\xi}\right\|_{-\tau, s, \infty}^{2 \alpha}+|Z(0)|^{2}\right) .
\end{aligned}
$$

For $J_{3}$, we can estimate

$$
\begin{aligned}
\left|J_{3}(s)\right| & \leq C_{4} s^{H-\frac{1}{2}} \int_{0}^{s} \frac{\left\|Y_{s}^{\xi}-Y_{r}^{\xi}\right\|_{\infty}^{\alpha} \wedge\left(1+C_{5}\left(\left\|Y_{s}^{\xi}\right\|_{\infty}^{p}+\left\|Y_{r}\right\|_{\infty}^{p}\right)\right)}{(s-r)^{H+1 / 2} r^{H-1 / 2}} \mathrm{~d} r \\
& \leq 2 C_{4} s^{H-\frac{1}{2}} \int_{0}^{s} \frac{\left(1+C_{5}\left\|Y^{\xi}\right\|_{-\tau, s, \infty}^{p}\right) \wedge\left(\left\|Y^{\xi}\right\|_{-\tau, s, \theta}^{\alpha}(s-r)^{\theta \alpha}\right)}{(s-r)^{H+1 / 2} r^{H-1 / 2}} \mathrm{~d} r \\
& \leq 2 C_{4} s^{H-\frac{1}{2}}\left(1+C_{5}\left\|Y^{\xi}\right\|_{-\tau, s, \infty}^{p}\right) \int_{0}^{s} \frac{\left\|Y^{\xi}\right\|_{-\tau, s, \theta}^{\alpha}(s-r)^{\theta \alpha-H-1 / 2} r^{-H+1 / 2}}{1+C_{5}\left\|Y^{\xi}\right\|_{-\tau, s, \infty}^{p}+\left\|Y^{\xi}\right\|_{-\tau, s, \theta}^{\alpha}(s-r)^{\theta \alpha}} \mathrm{d} r \\
& \leq 2 C_{4} s^{H-1 / 2}\left(1+C_{5}\left\|Y^{\xi}\right\|_{-\tau, s, \infty}^{p}\right)
\end{aligned}
$$




$$
\times\left(\frac{2^{2 H-1} s^{1-2 H}}{3 / 2-H}+\frac{\theta \alpha 2^{H-1 / 2} s^{\frac{1}{2}-H}\left\|Y^{\xi}\right\|_{-\tau, s, \theta}^{\frac{H-1 / 2}{\theta}}\left(1+C_{5}\left\|Y^{\xi}\right\|_{-\tau, s, \infty}^{p}\right)^{-\frac{H-1 / 2}{\theta \alpha}}}{(\alpha \theta-H+1 / 2)(H-1 / 2)}\right),
$$

where we used [5, Lemma 3.4] in the last inequality. Thus

$$
\begin{aligned}
\left|J_{3}(s)\right|^{2} & \leq C_{6} s^{1-2 H}\left(1+C_{5}\left\|Y^{\xi}\right\|_{-\tau, s, \infty}^{p}\right)^{2} \\
& +C_{7}\left(1+C_{5}\left\|Y^{\xi}\right\|_{-\tau, s, \infty}^{p}\right)^{\frac{2 \theta \alpha-2 H+1}{\theta \alpha}}\left\|Y^{\xi}\right\|_{-\tau, s, \theta}^{\frac{2 H-1}{\theta}}
\end{aligned}
$$

where

$$
C_{6}=8 C_{4}^{2}, \quad C_{7}=\left(\frac{\theta \alpha 2^{H}}{(\alpha \theta-H+1 / 2)(H-1 / 2)}\right)^{2} .
$$

Since $\left\|Y^{\xi}\right\|_{-\tau, s, \infty} \leq(s+\tau)^{\theta}\left\|Y^{\xi}\right\|_{-\tau, s, \theta}$, it follows from (4.14) and (4.15) that

$$
\begin{aligned}
& \mathbb{E} \exp \left\{C \int_{0}^{T}\left|K_{H}^{-1}\left(\int_{0}^{\cdot} Z\left(Y_{r}^{\xi}\right) \mathrm{d} r\right)(s)\right|^{2} \mathrm{~d} s\right\} \\
& \leq \mathbb{E} \exp \left(C_{T}\left(1+\left\|Y^{\xi}\right\|_{-\tau, s, \theta}^{2 p \vee \frac{2 \theta \alpha p+(2 H-1)(\alpha-p)}{\theta \alpha}}\right)\right) \\
& =\mathbb{E} \exp \left(C_{T}\left(1+\left\|Y^{\xi}\right\|_{-\tau, s, \theta}^{2 p+\frac{(2 H-1)(\alpha-p)}{\theta \alpha}}\right)\right) .
\end{aligned}
$$

For $p<1$, it is clear that

$$
2 p+\frac{(2 H-1)(\alpha-p)^{+}}{\theta \alpha}<2 .
$$

Then (4.6) follows from (4.3) with $q_{0}=1, \beta=\theta$ and the Fernique-type lemma.

(3) For $p=1$, substituting (4.14) and (4.15) into (4.8), we have

$$
\begin{aligned}
\int_{0}^{T} \mid & \left.K_{H}^{-1}\left(\int_{0} Z\left(Y_{r}^{\xi}\right) \mathrm{d} r\right)\right|^{2}(s) \mathrm{d} s \leq C_{8}(T)+\frac{3 C_{4}^{2} T^{2-2 H}\left(1+(H-1 / 2)^{2} C_{0}^{2}\right)}{(1-H) \Gamma^{2}(3 / 2-H)}\left\|Y^{\xi}\right\|_{-\tau, T, \infty}^{2 \alpha} \\
& +\frac{3 C_{6} C_{5}^{2} T^{2-2 T}(H-1 / 2)^{2}}{(1-H) \Gamma^{2}(3 / 2-H)}\left\|Y^{\xi}\right\|_{-\tau, T, \infty}^{2}+\frac{3 C_{7} T^{2-2 H}(H-1 / 2)^{2}}{2(1-H) \Gamma^{2}(3 / 2-H)}\left\|Y^{\xi}\right\|_{-\tau, T, \theta}^{\frac{2 H-1}{\theta}} \\
& +\frac{3 C_{7} T^{2(1-H+\theta)-\frac{2 H-1}{\alpha}}(H-1 / 2)^{2}}{2(1-H) \Gamma^{2}(3 / 2-H)}\left\|Y^{\xi}\right\|_{-\tau, T, \theta}^{2+\frac{2 H-1}{\theta}\left(1-\alpha^{-1}\right)},
\end{aligned}
$$

where

$$
C_{8}(T)=\frac{3 T^{2-2 H}\left(1+(H-1 / 2)^{2} C_{0}^{2}\right)}{(1-H) \Gamma^{2}(3 / 2-H)}\left(|Z(0)|^{2}+C_{6}(H-1 / 2)^{2}\right) .
$$

It follows from (4.1) and (A2) with $q_{0}=1$, we have

$$
\left\|Y^{\xi}\right\|_{0, T, \infty} \leq \widetilde{C}(T)+\left(L_{1} \Phi\left(\bar{K}_{1}, \bar{K}_{2}, T\right)+1\right)\|\sigma\|\left\|B^{H}\right\|_{0, T, \infty} .
$$


Therefore, for $T>0$ such that (4.7) holds, it follows from Lemma 2.1 that there is some $C>1$ such that

$$
\mathbb{E} \exp \left\{C \int_{0}^{T}\left|K_{H}^{-1}\left(\int_{0}^{\cdot} Z\left(Y_{r}^{\xi}\right) \mathrm{d} r\right)(s)\right|^{2} \mathrm{~d} s\right\}<\infty
$$

\section{Proof of Theorem 3.1}

We first show the existence of weak solution to (3.1). It follows from (A1)-(A3) and Lemma 4.2 that $R^{\xi}(t)$ is an exponential martingale. Then the Girsanov theorem implies that $\widetilde{B}^{H}(t)$ is a fBM under $\mathbb{Q}^{\xi}:=R^{\xi}(T) \mathbb{P}$. Reformulating the reference $\operatorname{SDE}(3.2)$ as follows:

$$
\mathrm{d} Y^{\xi}(t)=b\left(Y^{\xi}(t)\right) \mathrm{d} t+\sigma Z\left(Y_{t}^{\xi}\right) \mathrm{d} t+\sigma \mathrm{d} \widetilde{B}^{H}(t)
$$

then under the complete filtration probability $\left(\Omega, \mathscr{F},\left(\mathscr{F}_{t}\right)_{t \in[0, T]}, \mathbb{Q}^{\xi}\right),\left(Y^{\xi}(t), \widetilde{B}^{H}(t)\right)_{t \in[0, T]}$ is a solution of (3.1).

We shall show the uniqueness of weak solutions to (3.1), see [29, Theorem 2.1] for more proof details. We sketch the proof as follows:

For $i=1,2$, let $\left(Y^{(i), \xi}(t), B_{i}^{H}(t)\right)_{t \in[0, T]}$ be two weak solutions to (3.1) under the complete filtration probability space $\left(\Omega^{(i)},\left\{\mathscr{F}_{t}^{(i)}\right\}_{t \geq 0}, \mathbb{P}^{(i)}\right)$ with $Y_{0}^{(i), \xi}=\xi$ satisfying (3.9). Note that $Y^{\xi}(\cdot) \in C^{\beta}\left([0, T], \mathbb{R}^{d}\right)$ for any $\beta \in(0, H)$. Since $\xi \in C^{\theta}\left([-\tau, 0], \mathbb{R}^{d}\right)$, we obtain $Y^{\xi} \in$ $C^{\theta \wedge \beta}([0, T], \mathscr{C})$. Let $\beta>\frac{2 H-1}{2 \alpha}$. Then $(\theta \wedge \beta) \alpha+\frac{1}{2}-H>0$, which ensure the integrals in (4.8) make sense. By (4.11), (4.12) and (4.13), we have

$$
\int_{0}^{t}\left|K_{H}^{-1}\left(\int_{0}^{\cdot} Z\left(Y_{s}^{(i), \xi}\right) \mathrm{d} s\right)\right|^{2}(r) \mathrm{d} r<\infty, t \in[0, T], \mathbb{P}-\text { a.s. }
$$

Denote by $\mathbb{P}^{(i), \xi}$ the distribution of $Y^{(i), \xi}$, which satisfies

$$
\mathbb{P}^{(i), \xi}\left(Y^{(i), \xi}(\cdot) \in C\left([0, T] ; \mathbb{R}^{d}\right): \int_{0}^{T}\left|K_{H}^{-1}\left(\int_{0} Z\left(Y_{s}^{(i), \xi}\right) \mathrm{d} s\right)\right|^{2}(r) \mathrm{d} r<\infty, T>0\right)=1,
$$

we intend to prove $\mathbb{P}^{(1), \xi}=\mathbb{P}^{(2), \xi}$. To this end, we define

$$
\tau_{n}^{(i)}:=\inf \left\{t \geq 0: \int_{0}^{t}\left|K_{H}^{-1}\left(\int_{0}^{\cdot} Z\left(Y_{s}^{(i), \xi}\right) \mathrm{d} s\right)\right|^{2}(r) \mathrm{d} r \geq n\right\} \uparrow \infty, \text { as } n \uparrow \infty, i=1,2 .
$$

For every $i=1,2$ and $n \geq 1$,

$$
R_{n}^{(i), \xi}(t):=\exp \left\{-\int_{0}^{t}\left\langle K_{H}^{-1}\left(\int_{0}^{\cdot} Z\left(Y_{s}^{(i), \xi}\right) \mathrm{d} s\right)(r), \mathrm{d} B_{i}(r)\right\rangle-\frac{1}{2} \int_{0}^{t}\left|K_{H}^{-1}\left(\int_{0}^{\cdot} Z\left(Y_{s}^{(i), \xi}\right) \mathrm{d} s\right)\right|^{2}(r) \mathrm{d} r\right\}
$$

is a $\mathbb{P}^{(i), \xi}$-martingale. Define the probability measure $\mathbb{Q}_{n}^{(i), \xi}$ on $\mathscr{F}_{\infty}^{(i), \xi}$ by letting

$$
\mathbb{Q}_{n}^{(i), \xi}(A)=\mathbb{E}_{\mathbb{P}^{(i), \xi}}\left[I_{A} R_{n}^{(i), \xi}(T)\right], \quad T>0, A \in \mathscr{F}_{T}^{(i), \xi}
$$


By the Girsanov theorem, we know

$$
\widehat{B}_{i}^{H}(t):=B_{i}^{H}(t)+\int_{0}^{t \wedge \tau_{n}^{(i)}} Z\left(Y_{s}^{(i), \xi}\right) \mathrm{d} s, t \geq 0
$$

is a $\mathbb{Q}_{n}^{(i)}$-fractional Brownian motion on $\mathbb{R}^{m}$.

Therefore, under the $\mathbb{Q}_{n}^{(i)},\left(Y^{(i), \xi}(t), \widehat{B}_{i}^{H}(t)\right)_{t \in\left[0, T \wedge \tau_{n}^{\xi}\right]}$ solves (3.2) under the $\mathbb{Q}_{n}^{(i)}$. By the pathwise uniqueness of $(3.2)$, the law of $\left(Y^{(i), \xi}(t), \widehat{B}_{i}^{H}(t)\right)_{t \in\left[0, T \wedge \tau_{n}^{(i)}\right]}$ under $\mathbb{Q}_{n}^{(i)}$ coincides with the law of $\left(Y^{\xi}(t), B^{H}(t)\right)_{t \in\left[0, T \wedge \tau_{n}^{\xi}\right]}$ under $\mathbb{P}$. Thus, for any $F \in \mathscr{B}_{b}\left(C\left([0, T] ; \mathbb{R}^{d}\right) \times\right.$ $\left.C\left([0, T] ; \mathbb{R}^{d}\right)\right)$, we have

$$
\begin{aligned}
\left.\mathbb{E}_{\mathbb{P}^{(i)}}\left[I_{\left\{\tau_{n}^{(i)} \geq T\right\}} F\left(Y^{(i), \xi}(t), B_{i}^{H}(t)\right)\right)\right] \\
=\mathbb{E}_{\mathbb{Q}_{n}^{(i)}}\left[I _ { \{ \int _ { 0 } ^ { T } | K _ { H } ^ { - 1 } ( \int _ { 0 } Z ( Y _ { s } ^ { ( i ) , \xi } ) \mathrm { d } s ) | ^ { 2 } ( r ) \mathrm { d } r \leq n \} } \operatorname { e x p } \left\{\int_{0}^{T}\left\langle K_{H}^{-1}\left(\int_{0} Z\left(Y_{s}^{(i), \xi}\right) \mathrm{d} s\right)(r), \mathrm{d} B_{i}(r)\right\rangle\right.\right. \\
\left.-\frac{1}{2} \int_{0}^{t}\left|K_{H}^{-1}\left(\int_{0} Z\left(Y_{s}^{(i), \xi}\right) \mathrm{d} s\right)\right|^{2}(r) \mathrm{d} r\right\} \\
\left.\quad \times F\left(Y^{(i), \xi}([0, T]),\left(\widehat{B}_{i}^{H}-\int_{0} Z\left(Y_{s}^{(i), \xi}\right) \mathrm{d} s\right)([0, T])\right)\right] \\
=\mathbb{E}_{\mathbb{P}}\left[I _ { \{ \int _ { 0 } ^ { T } | K _ { H } ^ { - 1 } ( \int _ { 0 } ^ { \cdot } Z ( Y _ { s } ^ { \xi } ) \mathrm { d } s ) | ^ { 2 } ( r ) \mathrm { d } r \leq n \} } \operatorname { e x p } \left\{\int_{0}^{T}\left\langle K_{H}^{-1}\left(\int_{0} Z\left(Y_{s}^{\xi}\right) \mathrm{d} s\right)(r), \mathrm{d} B(r)\right\rangle\right.\right. \\
\left.-\frac{1}{2} \int_{0}^{t}\left|K_{H}^{-1}\left(\int_{0} Z\left(Y_{s}^{\xi}\right) \mathrm{d} s\right)\right|^{2}(r) \mathrm{d} r\right\} \\
\left.\quad \times F\left(Y^{\xi}([0, T]),\left(B^{H}-\int_{0} Z\left(Y_{s}^{\xi}\right) \mathrm{d} s\right)([0, T])\right)\right], \quad i=1,2 .
\end{aligned}
$$

Consequently,

$$
\mathbb{E}_{\mathbb{P}^{(1)}}\left[I_{\left\{\tau_{n}^{(1)} \geq T\right\}} F\left(Y^{(1), \xi}([0, T]), B_{1}^{H}([0, T])\right)\right]=\mathbb{E}_{\mathbb{P}^{(2)}}\left[I_{\left\{\tau_{n}^{(2)} \geq T\right\}} F\left(Y^{(2), \xi}([0, T]), B_{2}^{H}([0, T])\right)\right],
$$

holds for any $n \geq 1$. Letting $n \rightarrow \infty$, we obtain

$$
\mathbb{E}_{\mathbb{P}^{(1)}}\left[F\left(Y^{(1), \xi}([0, T]), B_{1}^{H}([0, T])\right)\right]=\mathbb{E}_{\mathbb{P}^{(2)}}\left[F\left(Y^{(2), \xi}([0, T]), B_{2}^{H}([0, T])\right)\right],
$$

which implies that $\mathbb{P}^{(1)}=\mathbb{P}^{(2)}$. Thus, the uniqueness of weak solution to (3.1) is verified.

\section{$5 \quad$ Proof of Theorem 3.2}

Before giving the proof for Theorem 3.2, we prepare two lemmas. The lemma below shows the estimates of uniform norm and Hölder norm of $\left(Y^{\xi}(t)\right)_{t \in[0, T]}$ of the solution to (3.2), respectively. 
Lemma 5.1. Assume (H1). Then for any $T>0$

$$
\begin{aligned}
\left\|Y^{\xi}\right\|_{-\tau, T, \infty} \leq & \|\xi\|_{\infty}+|b(0)| \Phi\left(\bar{K}_{1}, \bar{K}_{2}, T\right)+\left(L_{1} \Phi\left(\bar{K}_{1}, \bar{K}_{2}, T\right)+1\right)\|\sigma\|\left\|B^{H}\right\|_{\infty} . \\
\left\|Y^{\xi}\right\|_{-\tau, T, \beta \wedge \theta} \leq & T^{1-\beta \wedge \theta}\left(|b(0)|+|b(0)| L_{1} \Phi\left(\bar{K}_{1}, \bar{K}_{2}, T\right)+|\xi(0)|\right)+\|\sigma\|\left\|B^{H}\right\|_{\beta \wedge \theta} \\
& +L_{1} T^{1-\beta \wedge \theta}\left(L_{1} \Phi\left(\bar{K}_{1}, \bar{K}_{2}, T\right)+1\right)\|\sigma\|\left\|B^{H}\right\|_{\infty}+\|\xi\|_{-\tau, 0, \beta \wedge \theta} .
\end{aligned}
$$

Proof. The first inequality follows from (4.1) and (H1) directly. Since $b$ is Lipschitz,

$$
|b(x)| \leq|b(0)|+L_{1}|x| .
$$

Taking into account the following inequality

$$
\left\|Y^{\xi}\right\|_{-\tau, T, \beta \wedge \theta} \leq\|\xi\|_{-\tau, 0, \beta \wedge \theta}+\left\|Y^{\xi}\right\|_{\beta \wedge \theta},
$$

the proof of the second inequality is similar to the second part of the proof of Lemma 4.1.

For the sake of simplicity, we denote

$$
h^{\xi}(t)=\widehat{\sigma}^{-1}\left\{b\left(Y^{\xi}(t)\right)-b\left(Y^{\xi}\left(t_{\delta}\right)\right\}-Z\left(\widehat{Y}_{t}^{\xi}\right), \quad t \geq 0,\right.
$$

with

$$
\widehat{Y}_{t}^{\xi}(u)=Y^{\xi}\left((t+u) \wedge t_{\delta}\right), \quad u \in[-\tau, 0] .
$$

Let

$$
\begin{aligned}
B_{h}^{H}(t)=B^{H}(t) & +\int_{0}^{t} h^{\xi}(s) \mathrm{d} s, \\
R^{\xi, \delta}(t)=\exp \{ & -\int_{0}^{t}\left\langle K_{H}^{-1}\left(\int_{0} h^{\xi}(s) \mathrm{d} s\right)(r), \mathrm{d} B(r)\right\rangle \\
& \left.-\frac{1}{2} \int_{0}^{t}\left|K_{H}^{-1}\left(\int_{0} h^{\xi}(s) \mathrm{d} s\right)(r)\right|^{2} \mathrm{~d} r\right\}, t \in[0, T],
\end{aligned}
$$

and let $\mathrm{d}_{\mathbb{Q}}^{\xi, \delta}=R^{\xi, \delta}(T) \mathrm{d} \mathbb{P}$. Then it follows from Lemma 5.2 below and the Girsanov theorem that $\mathbb{Q}^{\xi, \delta}$ is a probability and $\left(B_{h}^{H}(t)\right)_{t \in[0, T]}$ is a fBM under $\mathbb{Q}^{\xi, \delta}$. Since $\sigma \sigma^{-1}=\pi_{*}$, we can rewrite the reference SDE (3.2) into the following form

$$
\mathrm{d} Y^{\xi}(t)=\left\{\left(I_{d \times d}-\pi_{*}\right) b\left(Y^{\xi}(t)\right)+\pi_{*} b\left(Y^{\xi}\left(t_{\delta}\right)\right)+\sigma Z\left(\widehat{Y}_{t}^{\xi}\right)\right\} \mathrm{d} t+\sigma \mathrm{d} B_{h}^{H}(t),
$$

which implies that $\left(Y^{\xi}(t), B_{h}^{H}(t)\right)_{t \in[0, T]}$ is a weak solution of (3.10). Obviously, (3.10) has a unique pathwise solution, so the weak uniqueness follows. Then

$$
\left|\mathbb{E} f(X(t))-\mathbb{E} f\left(X^{(\delta)}(t)\right)\right|=\left|\mathbb{E}_{\mathbb{Q}^{\xi}} f\left(Y^{\xi}(t)\right)-\mathbb{E}_{\mathbb{Q}^{\xi}, \delta} f\left(Y^{\xi}(t)\right)\right|=\left|\mathbb{E}\left(R^{\xi}(t)-R^{\xi, \delta}(t)\right) f\left(Y^{\xi}(t)\right)\right| .
$$

Hence, in the following discussion, we shall prove that $\left\{R^{\xi, \delta}(t)\right\}_{t \in[0, T]}$ is an exponential martingale and give estimates of $R^{\xi}(t)-R^{\xi, \delta}(t)$. 
Lemma 5.2. Under the assumptions of Theorem 3.2, we have

$$
\mathbb{E} \exp \left\{C \int_{0}^{t}\left|K_{H}^{-1}\left(\int_{0}^{\cdot} h^{\xi}(s) \mathrm{d} s\right)\right|^{2}(r) \mathrm{d} r\right\}<\infty,
$$

for some $C>1$.

Proof. The definition of inverse operator $K_{H}^{-1}$ yields that

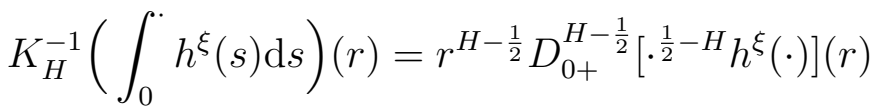

$$
\begin{aligned}
& =r^{H-\frac{1}{2}} \frac{1}{\Gamma\left(\frac{3}{2}-H\right)}\left(\frac{r^{\frac{1}{2}-H} h^{\xi}(r)}{r^{H-\frac{1}{2}}}+\left(H-\frac{1}{2}\right) \int_{0}^{r} \frac{r^{\frac{1}{2}-H} h^{\xi}(r)-s^{\frac{1}{2}-H} h^{\xi}(s)}{(r-s)^{H+\frac{1}{2}}} \mathrm{~d} s\right) \\
& =\frac{r^{\frac{1}{2}-H} h^{\xi}(r)}{\Gamma\left(\frac{3}{2}-H\right)}+\frac{r^{H-\frac{1}{2}}}{\Gamma\left(\frac{3}{2}-H\right)}\left(H-\frac{1}{2}\right) \int_{0}^{r} \frac{r^{\frac{1}{2}-H} h^{\xi}(r)-s^{\frac{1}{2}-H} h^{\xi}(s)}{(r-s)^{H+\frac{1}{2}}} \mathrm{~d} s \\
& =\frac{r^{\frac{1}{2}-H} h^{\xi}(r)}{\Gamma\left(\frac{3}{2}-H\right)}+\frac{r^{H-\frac{1}{2}}}{\Gamma\left(\frac{3}{2}-H\right)}\left(H-\frac{1}{2}\right) \int_{0}^{r} \frac{\left(r^{\frac{1}{2}-H}-s^{\frac{1}{2}-H}\right) h^{\xi}(r)}{(r-s)^{H+\frac{1}{2}}} \mathrm{~d} s \\
& +\frac{r^{H-\frac{1}{2}}}{\Gamma\left(\frac{3}{2}-H\right)}\left(H-\frac{1}{2}\right) \int_{0}^{r} \frac{s^{\frac{1}{2}-H}\left(h^{\xi}(r)-h^{\xi}(s)\right)}{(r-s)^{H+\frac{1}{2}}} \mathrm{~d} s \\
& =\left[1+C_{0}\left(H-\frac{1}{2}\right)\right] \frac{r^{\frac{1}{2}-H} h^{\xi}(r)}{\Gamma\left(\frac{3}{2}-H\right)}+\frac{r^{H-\frac{1}{2}}}{\Gamma\left(\frac{3}{2}-H\right)}\left(H-\frac{1}{2}\right) \int_{0}^{r} \frac{s^{\frac{1}{2}-H}\left(h^{\xi}(r)-h^{\xi}(s)\right)}{(r-s)^{H+\frac{1}{2}}} \mathrm{~d} s \\
& =: \widehat{J}_{1}(r)+\widehat{J}_{2}(r) \text {. }
\end{aligned}
$$

For $\widehat{J}_{1}$, it follows from $(\mathrm{H} 1)$ and $(\mathrm{H} 2)$ that

$$
\begin{aligned}
\left|h^{\xi}(r)\right| & \leq\left\|\widehat{\sigma}^{-1}\right\|\left|b\left(Y^{\xi}(r)\right)-b\left(Y^{\xi}\left(r_{\delta}\right)\right)\right|+\left|Z\left(\widehat{Y}_{r}^{\xi}\right)\right| \\
& \leq\left\|\widehat{\sigma}^{-1}\right\| L_{1}\left\|Y^{\xi}\right\|_{0, r, \beta} \delta^{\beta}+|Z(0)|+L_{2}\left\|Y^{\xi}\right\|_{-\tau, r, \infty}^{\alpha} .
\end{aligned}
$$

For $\widehat{J}_{2}$, note that

$$
\begin{aligned}
& \int_{0}^{r} \frac{s^{\frac{1}{2}-H}\left|h^{\xi}(r)-h^{\xi}(s)\right|}{(r-s)^{H+\frac{1}{2}}} \mathrm{~d} s \\
= & \int_{0}^{r} \frac{\left.s^{\frac{1}{2}-H} \mid \widehat{\sigma}^{-1}\left(b\left(Y^{\xi}(r)\right)-b\left(Y^{\xi}\left(r_{\delta}\right)\right)\right)-Z\left(\widehat{Y}_{r}^{\xi}\right)-\widehat{\sigma}^{-1}\left(b\left(Y^{\xi}(s)\right)-b\left(Y^{\xi}\left(s_{\delta}\right)\right)\right)+Z\left(\widehat{Y}_{s}^{\xi}\right)\right) \mid}{(r-s)^{H+\frac{1}{2}}} \mathrm{~d} s \\
\leq & \int_{0}^{r} \frac{\| \widehat{\sigma}^{-1}|| s^{\frac{1}{2}-H}\left|b\left(Y^{\xi}(r)\right)-b\left(Y^{\xi}\left(r_{\delta}\right)\right)-\left(b\left(Y^{\xi}(s)\right)-b\left(Y^{\xi}\left(s_{\delta}\right)\right)\right)\right|}{(r-s)^{H+\frac{1}{2}}} \mathrm{~d} s \\
& +\int_{0}^{r} \frac{\left.s^{\frac{1}{2}-H} \mid Z\left(\widehat{Y}_{r}^{\xi}\right)-Z\left(\widehat{Y}_{s}^{\xi}\right)\right) \mid}{(r-s)^{H+\frac{1}{2}}} \mathrm{~d} s \\
= & : I_{1}(r)+I_{2}(r) .
\end{aligned}
$$


Next, we shall give the estimate of $I_{i}(r), i=1,2$. For $I_{1}(r)$, it follows from $(\mathrm{H} 1)$ that

$$
\begin{aligned}
& \left|b\left(Y^{\xi}(r)\right)-b\left(Y^{\xi}\left(r_{\delta}\right)\right)-\left(b\left(Y^{\xi}(s)\right)-b\left(Y^{\xi}\left(s_{\delta}\right)\right)\right)\right| \\
& \leq 2 L_{1}\left\|Y^{\xi}\right\|_{0, r, \beta}\left[\delta^{\beta} \wedge \frac{(r-s)^{\beta}+\left(r_{\delta}-s_{\delta}\right)^{\beta}}{2}\right] \\
& \quad=L_{1}\left\|Y^{\xi}\right\|_{0, r, \beta}\left\{\begin{array}{lr}
(r-s)^{\beta}, & r_{\delta}<s<r, \\
(r-s)^{\beta}+\left(r_{\delta}-s_{\delta}\right)^{\beta}, & r-\delta<s<r_{\delta}, \\
2 \delta^{\beta}, & 0<s<r-\delta .
\end{array}\right.
\end{aligned}
$$

Since

$$
\left|r_{\delta}-s_{\delta}\right|=\left|\left[\frac{r}{\delta}\right] \delta-\left[\frac{s}{\delta}\right] \delta\right| \leq\left|\left[\frac{r}{\delta}\right] \delta-\left[\frac{r-\delta}{\delta}\right] \delta\right| \leq \delta, \quad r-\delta<s<r_{\delta},
$$

and for $r \geq \delta$

$$
\begin{aligned}
& \int_{r_{\delta}}^{r} \frac{s^{1 / 2-H}}{(r-s)^{H+1 / 2-\beta}} \mathrm{d} s \leq \frac{2 \delta^{1 / 2+\beta-H}}{1+2 \beta-2 H} r_{\delta}^{1 / 2-H}, \\
& \int_{0}^{r_{\delta}} \frac{2 \delta^{\beta}}{(r-s)^{H+1 / 2} s^{H-1 / 2}} \mathrm{~d} s \\
& =\int_{r_{\delta} / 2}^{r_{\delta}} \frac{2 \delta^{\beta}}{(r-s)^{1 / 2+H} s^{H-1 / 2}} \mathrm{~d} s+\int_{0}^{r_{\delta} / 2} \frac{2 \delta^{\beta}}{(r-s)^{1 / 2+H} s^{H-1 / 2}} \mathrm{~d} s \\
& \leq \frac{2 \delta^{\beta}\left(r-r_{\delta}\right)^{\frac{1}{2}-H}}{\left(r_{\delta} / 2\right)^{H-1 / 2}(H-1 / 2)}+\frac{2 \delta^{\beta}\left(\frac{r_{\delta}}{2}\right)^{\frac{3}{2}-H}}{\left(r-r_{\delta} / 2\right)^{H+1 / 2}\left(\frac{3}{2}-H\right)},
\end{aligned}
$$

we have

$$
\begin{aligned}
\left|I_{1}(r)\right| & \leq 2 L_{1}\left\|\widehat{\sigma}^{-1}\right\|\left\|Y^{\xi}\right\|_{0, r, \beta}\left\{\left[\frac{\delta^{\beta+1 / 2-H}}{(2 \beta+1-2 H) r_{\delta}^{H-1 / 2}}+\frac{\delta^{\beta}\left(r-r_{\delta}\right)^{\frac{1}{2}-H}}{\left(r_{\delta} / 2\right)^{H-1 / 2}(H-1 / 2)}\right.\right. \\
& \left.\left.+\frac{\delta^{\beta}\left(\frac{r_{\delta}}{2}\right)^{\frac{3}{2}-H}}{\left(r-r_{\delta} / 2\right)^{H+1 / 2}\left(\frac{3}{2}-H\right)}\right] \mathbb{1}_{[r \geq \delta]}+\frac{1}{2} \mathcal{B}\left(\frac{3}{2}-H, \beta+\frac{1}{2}-H\right) r^{\beta+1-2 H} \mathbb{1}_{[0 \leq r<\delta]}\right\} .
\end{aligned}
$$

We now calculate $I_{2}(r)$. One can see that

$$
\begin{aligned}
& \left\|\widehat{Y}_{r}^{\xi}-\widehat{Y}_{s}^{\xi}\right\|_{\infty}^{\alpha} \\
& =\sup _{-\tau \leq u \leq 0} \frac{\left|Y^{\xi}\left((r+u) \wedge r_{\delta}\right)-Y^{\xi}\left((s+u) \wedge s_{\delta}\right)\right|^{\alpha}}{\left|(r+u) \wedge r_{\delta}-(s+u) \wedge s_{\delta}\right|^{\alpha(\beta \wedge \theta)}}\left|(r+u) \wedge r_{\delta}-(s+u) \wedge s_{\delta}\right|^{\alpha(\beta \wedge \theta)} \\
& \leq\left\|Y^{\xi}\right\|_{-\tau, r, \beta \wedge \theta}^{\alpha} \sup _{-\tau \leq u \leq 0}\left|(r+u) \wedge r_{\delta}-(s+u) \wedge s_{\delta}\right|^{\alpha(\beta \wedge \theta)} .
\end{aligned}
$$

Since for $s+u>s_{\delta}$ and $r+u<r_{\delta}$, we have

$$
(s+u) \wedge s_{\delta}=s_{\delta} ; \quad(r+u) \wedge r_{\delta}=r+u ; \quad s_{\delta}-s<u<r_{\delta}-r .
$$


Then

$$
\sup _{s_{\delta}-s<u<r_{\delta}-r}\left|(r+u) \wedge r_{\delta}-(s+u) \wedge s_{\delta}\right|=\sup _{s_{\delta}-s<u<r_{\delta}-r}\left|r+u-s_{\delta}\right|=\left|r_{\delta}-s_{\delta}\right| .
$$

Similarly, for $s+u<s_{\delta}$ and $r+u>r_{\delta}$, we have

$$
\sup _{r_{\delta}-r<u<s_{\delta}-s}\left|(r+u) \wedge r_{\delta}-(s+u) \wedge s_{\delta}\right|=\left|r_{\delta}-s_{\delta}\right|
$$

Then it is easy to see that

$$
\sup _{u \in[-\tau, 0]}\left|(r+u) \wedge r_{\delta}-(s+u) \wedge s_{\delta}\right|=(r-s) \vee\left(r_{\delta}-s_{\delta}\right)
$$

Consequently

$$
\left\|\widehat{Y}_{r}^{\xi}-\widehat{Y}_{s}^{\xi}\right\|^{\alpha} \leq\left\|Y^{\xi}\right\|_{-\tau, r, \beta \wedge \theta}^{\alpha}\left((r-s) \vee\left(r_{\delta}-s_{\delta}\right)\right)^{\alpha(\beta \wedge \theta)}
$$

and

$$
\begin{aligned}
I_{2}(r) & =\int_{0}^{r} \frac{\left.s^{\frac{1}{2}-H} \mid Z\left(\widehat{Y}_{r}^{\xi}\right)-Z\left(\widehat{Y}_{s}^{\xi}\right)\right) \mid}{(r-s)^{H+\frac{1}{2}}} \mathrm{~d} s \\
& \leq \int_{0}^{r} \frac{L_{2}\left\|\widehat{Y}_{r}^{\xi}-\widehat{Y}_{s}^{\xi}\right\|_{\infty}^{\alpha}}{(r-s)^{1 / 2+H} s^{H-1 / 2}} \mathrm{~d} s \\
& \leq L_{2}\|Y\|_{-\tau, r, \beta \wedge \theta}^{\alpha} \int_{0}^{r} \frac{(r-s)^{\alpha(\beta \wedge \theta)} \vee\left(r_{\delta}-s_{\delta}\right)^{\alpha(\beta \wedge \theta)}}{(r-s)^{1 / 2+H} s^{H-1 / 2}} \mathrm{~d} s .
\end{aligned}
$$

Since $r_{\delta}-s_{\delta}=0$ for $s \in\left[r_{\delta}, r\right]$,

$$
\begin{aligned}
\int_{0}^{r} \frac{(r-s)^{\alpha(\beta \wedge \theta)} \vee\left(r_{\delta}-s_{\delta}\right)^{\alpha(\beta \wedge \theta)}}{(r-s)^{1 / 2+H} s^{H-1 / 2}} \mathrm{~d} s & =\int_{0}^{r} \frac{(r-s)^{\alpha(\beta \wedge \theta)}}{(r-s)^{1 / 2+H} s^{H-1 / 2}} \mathbf{1}_{\left[r-s \geq r_{\delta}-s_{\delta}\right]} \mathrm{d} s \\
& +\int_{0}^{r_{\delta}} \frac{\left(r_{\delta}-s_{\delta}\right)^{\alpha(\beta \wedge \theta)}}{(r-s)^{1 / 2+H} s^{H-1 / 2}} \mathbf{1}_{\left[r-s<r_{\delta}-s_{\delta}\right]} \mathrm{d} s .
\end{aligned}
$$

For $r-r_{\delta}+s_{\delta}<s$, it is clear that $r_{\delta}-s_{\delta}-(r-s) \leq \delta$, so

$$
\left(r_{\delta}-s_{\delta}\right)^{\alpha(\beta \wedge \theta)}=\left(r_{\delta}-s_{\delta}-r+s+(r-s)\right)^{\alpha(\beta \wedge \theta)} \leq(r-s)^{\alpha(\beta \wedge \theta)}+\delta^{\alpha(\beta \wedge \theta)},
$$

which implies that

$$
\int_{0}^{r_{\delta}} \frac{\left(r_{\delta}-s_{\delta}\right)^{\alpha(\beta \wedge \theta)}}{(r-s)^{1 / 2+H} s^{H-1 / 2}} \mathbf{1}_{\left[r-s<r_{\delta}-s_{\delta}\right]} \mathrm{d} s \leq \int_{0}^{r_{\delta}} \frac{(r-s)^{\alpha(\beta \wedge \theta)}+\delta^{\alpha(\beta \wedge \theta)}}{(r-s)^{1 / 2+H} s^{H-1 / 2}} \mathbf{1}_{\left[r-s<r_{\delta}-s_{\delta}\right]} \mathrm{d} s .
$$

Moreover, we have

$$
\int_{0}^{T}\left(\int_{0}^{r_{\delta}} \frac{\delta^{\alpha(\beta \wedge \theta)} \mathbf{1}_{\left[r-s<r_{\delta}-s_{\delta}\right]}}{(r-s)^{1 / 2+H} s^{H-1 / 2}} \mathrm{~d} s\right)^{2} \mathrm{~d} r \leq \int_{\delta}^{T}\left(\int_{0}^{r_{\delta}} \frac{\delta^{\alpha(\beta \wedge \theta)}}{(r-s)^{1 / 2+H} s^{H-1 / 2}} \mathrm{~d} s\right)^{2} \mathrm{~d} r
$$




$$
\begin{aligned}
& \leq \sum_{k=1}^{N-1} \int_{k \delta}^{(k+1) \delta}\left(\frac{\delta^{\alpha(\beta \wedge \theta)}\left(\frac{r_{\delta}}{2}\right)^{\frac{3}{2}-H}}{\left(r-r_{\delta} / 2\right)^{H+1 / 2}\left(\frac{3}{2}-H\right)}+\frac{\delta^{\alpha(\beta \wedge \theta)}\left(r-r_{\delta}\right)^{\frac{1}{2}-H}}{\left(r_{\delta} / 2\right)^{H-1 / 2}(H-1 / 2)}\right)^{2} \mathrm{~d} r \\
& \leq 2 \delta^{2 \alpha(\beta \wedge \theta)} \sum_{k=1}^{N-1}\left(\frac{16^{H}(k \delta)^{2-4 H} \delta}{(3-2 H)^{2}}+\frac{2^{2 H-1} \delta^{2-2 H}}{(H-1 / 2)^{2}(2-2 H)(k \delta)^{2 H-1}}\right) \\
& \leq 2 \delta^{2 \alpha(\beta \wedge \theta)+1-2 H}\left(\frac{16^{H}}{(3-2 H)^{2}}+\frac{2^{2 H}}{(2 H-1)^{2}(1-H)}\right) \sum_{k=1}^{N-1}(k \delta)^{1-2 H} \delta \\
& \leq \frac{\delta^{2 \alpha(\beta \wedge \theta)+1-2 H}}{1-H}\left(\frac{16^{H}}{(3-2 H)^{2}}+\frac{2^{2 H}}{(2 H-1)^{2}(1-H)}\right) T^{2-2 H}
\end{aligned}
$$

and

$$
\int_{0}^{T}\left(\int_{0}^{r} \frac{(r-s)^{\alpha(\beta \wedge \theta)}}{(r-s)^{1 / 2+H} s^{H-1 / 2}} \mathrm{~d} s\right)^{2} \mathrm{~d} r=\frac{T^{2 \alpha(\beta \wedge \theta)+3-4 H} \mathcal{B}^{2}\left(\frac{3}{2}-H, \alpha(\beta \wedge \theta)+\frac{1}{2}-H\right)}{2 \alpha(\beta \wedge \theta)+3-4 H} .
$$

Substituting $\widehat{J}_{1}, I_{1}(r)$ and $I_{2}(r)$ into (5.5), and taking into account (5.6) and (5.7), we arrive at

$$
\begin{aligned}
\int_{0}^{T} \mid K_{H}^{-1} & \left.\left(\int_{0}^{\cdot} h^{\xi}(s) \mathrm{d} s\right)(r)\right|^{2} \mathrm{~d} r \\
\leq & \frac{2 L_{1}^{2}\left\|\widehat{\sigma}^{-1}\right\|^{2}\left\|Y^{\xi}\right\|_{\beta}^{2}}{\Gamma^{2}\left(\frac{3}{2}-H\right)(1-H)}\left\{3\left[1+C_{0}\left(H-\frac{1}{2}\right)\right]^{2} T^{2-2 H} \delta^{2 \beta}\right. \\
& +2(2 H-1)^{2} T \delta^{2 \beta+1-2 H}\left[\frac{1}{(1+2 \beta-2 H)^{2}}+\frac{2^{4 H-1}}{(3-2 H)^{2}}+\frac{2^{2 H-1}}{(2 H-1)^{2}(1-H)}\right. \\
& \left.\left.+\frac{\mathcal{B}^{2}\left(\frac{3}{2}-H, \beta+\frac{1}{2}-H\right)(1-H) \delta}{8(1+\beta-H) T}\right]\right\} \\
& +\frac{6\left[1+C_{0}\left(H-\frac{1}{2}\right)\right]^{2} T^{2(1-H)}}{\Gamma^{2}\left(\frac{3}{2}-H\right)(1-H)}\left(|Z(0)|^{2}+L_{2}^{2}\left\|Y^{\xi}\right\|_{-\tau, T, \infty}^{2 \alpha}\right) \\
& +\frac{2 L_{2}^{2}(2 H-1)^{2} T^{2 H-1}\left\|Y^{\xi}\right\|_{-\tau, T, \beta \wedge \theta}^{2 \alpha}}{\Gamma^{2}\left(\frac{3}{2}-H\right)}\left[\frac{\mathcal{B}^{2}\left(\frac{3}{2}-H, \alpha(\beta \wedge \theta)+1 / 2-H\right) T^{2 \alpha(\beta \wedge \theta)+3-4 H}}{2 \alpha(\beta \wedge \theta)+3-4 H}\right. \\
& \left.+\frac{\delta^{2 \alpha(\beta \wedge \theta)+1-2 H} T^{2-2 H}}{1-H}\left(\frac{16^{H}}{(3-2 H)^{2}}+\frac{2^{2 H}}{(2 H-1)^{2}(1-H)}\right)\right] .
\end{aligned}
$$

Therefore, it follows from Lemma 5.1 and (3.12) that there exists $C>1$ such that

$$
\mathbb{E} \exp \left\{C \int_{0}^{T}\left|K_{H}^{-1}\left(\int_{0}^{\cdot} h^{\xi}(s) \mathrm{d} s\right)(r)\right|^{2} \mathrm{~d} r\right\}<\infty .
$$

We are now in the position to complete the 
Proof of Theorem 3.2. Let

$$
\begin{aligned}
& M_{1}(t)=\int_{0}^{t}\left\langle K_{H}^{-1}\left(\int_{0}^{\cdot} Z\left(Y_{s}^{\xi}\right) \mathrm{d} s\right)(r), \mathrm{d} B(r)\right\rangle, \\
& M_{2}(t)=\int_{0}^{t}\left\langle K_{H}^{-1}\left(\int_{0}^{\cdot} h^{\xi}(s) \mathrm{d} s\right)(r), d B(r)\right\rangle, \quad t \geq 0 .
\end{aligned}
$$

For $f \in \mathscr{B}_{b}\left(\mathbb{R}^{d}\right)$, following from the weak uniqueness of solution to (3.1), the Hölder inequality and the following inequality

$$
\left|\mathrm{e}^{x}-\mathrm{e}^{y}\right| \leq\left(\mathrm{e}^{x} \vee \mathrm{e}^{y}\right)|x-y|,
$$

we have

$$
\begin{aligned}
\left|\mathbb{E} f(X(t))-\mathbb{E} f\left(X^{(\delta)}(t)\right)\right| & =\left|\mathbb{E}_{\mathbb{Q}^{\xi}} f\left(Y^{\xi}(t)\right)-\mathbb{E}_{\mathbb{Q}^{\xi, \delta}} f\left(Y^{\xi}(t)\right)\right| \\
& =\left|\mathbb{E}\left(R^{\xi}(t)-R^{\xi, \delta}(t)\right) f\left(Y^{\xi}(t)\right)\right| \\
& \leq\|f\|_{\infty} \mathbb{E}\left|R^{\xi}(t)-R^{\xi, \delta}(t)\right| \\
& \leq\|f\|_{\infty} \mathbb{E}\left(R^{\xi}(t) \vee R^{\xi, \delta}(t)\right)\left|\log R^{\xi}(t)-\log R^{\xi, \delta}(t)\right| \\
& \leq\|f\|_{\infty} \Theta_{1}(t)\left(\Theta_{2}(t)+\Theta_{3}(t)\right), t \in[0, T],
\end{aligned}
$$

where

$\Theta_{1}(t)=\left(\mathbb{E}\left(R^{\xi}(t)\right)^{q}\right)^{\frac{1}{q}}+\left(\mathbb{E}\left(R^{\xi, \delta}(t)\right)^{q}\right)^{\frac{1}{q}}$,
$\Theta_{2}(t)=\left(\mathbb{E}\left|\int_{0}^{t}\left\langle K_{H}^{-1}\left(\int_{0}^{.}\left(Z\left(Y_{s}^{\xi}\right)+h^{\xi}(s)\right) \mathrm{d} s\right)(r), \mathrm{d} B(r)\right\rangle\right|^{\frac{q}{q-1}}\right)^{\frac{q-1}{q}}$,
$\Theta_{3}(t)=\frac{1}{2}\left(\mathbb{E}\left|\int_{0}^{t}\left(\left|K_{H}^{-1}\left(\int_{0}^{\cdot} Z\left(Y_{s}^{\xi}\right) \mathrm{d} s\right)(r)\right|^{2}-\left|K_{H}^{-1}\left(\int_{0}^{\cdot} h^{\xi}(s) \mathrm{d} s\right)(r)\right|^{2}\right) \mathrm{d} r\right|^{\frac{q}{q-1}}\right)^{\frac{q-1}{q}}, q>1$.

It follows from (3.11) and Lemma 4.2 with $C_{5}=0$ and $C_{4}=L_{2}$ that there is some $C>1$ such that $\mathbb{E} \exp \left\{C\left\langle M_{1}\right\rangle(T)\right\}<\infty$. Thus, for $2 q^{2}-q \leq C$, we have

$$
\begin{aligned}
\mathbb{E}\left(R^{\xi}(t)\right)^{q} & =\mathbb{E} \exp \left(q M_{1}(t)-q^{2}\left\langle M_{1}\right\rangle(t)+\left(q^{2}-q / 2\right)\left\langle M_{1}\right\rangle(t)\right) \\
& \leq\left(\mathbb{E} \exp \left(2 q M_{1}(t)-2 q^{2}\left\langle M_{1}\right\rangle(t)\right)\right)^{1 / 2}\left(\mathbb{E} \exp \left(\left(2 q^{2}-q\right)\left\langle M_{1}\right\rangle(t)\right)\right)^{1 / 2} \\
& \leq\left(\mathbb{E} \exp \left(\left(2 q^{2}-q\right) \int_{0}^{t}\left|K_{H}^{-1}\left(\int_{0} Z\left(Y_{s}^{\xi}\right) \mathrm{d} s\right)(r)\right|^{2} \mathrm{~d} r\right)\right)^{1 / 2} \\
& <\infty
\end{aligned}
$$

Similarly, following from Lemma 5.2, there is $q>1$ such that

$$
\sup _{t \in[0, T]}\left(\mathbb{E}\left(R^{\xi, \delta}(t)\right)^{q}\right)^{\frac{1}{q}}<\infty .
$$


Hence, there is $q>1$ and some constant $C_{T}$ such that

$$
\Theta_{1}(t) \leq C_{T} .
$$

In the following proof, we fix some $q>1$ such that (5.10) holds.

It is easy to see that

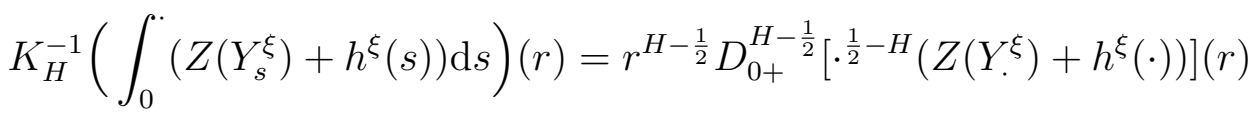

$$
\begin{aligned}
& =\frac{r^{H-\frac{1}{2}}}{\Gamma\left(\frac{3}{2}-H\right)}\left(\frac{r^{1 / 2-H}\left(Z\left(Y_{r}^{\xi}\right)+h^{\xi}(r)\right)}{r^{H-1 / 2}}\right. \\
& \left.+\left(H-\frac{1}{2}\right) \int_{0}^{r} \frac{r^{1 / 2-H}\left(Z\left(Y_{r}^{\xi}\right)+h^{\xi}(r)\right)-s^{1 / 2-H}\left(Z\left(Y_{s}^{\xi}\right)+h^{\xi}(s)\right)}{(r-s)^{H+1 / 2}} \mathrm{~d} s\right) \\
& \leq\left[1+C_{0}\left(H-\frac{1}{2}\right)\right] \frac{r^{\frac{1}{2}-H}\left(Z\left(Y_{r}^{\xi}\right)+h^{\xi}(r)\right)}{\Gamma\left(\frac{3}{2}-H\right)} \\
& +\frac{r^{H-\frac{1}{2}}}{\Gamma\left(\frac{3}{2}-H\right)}\left(H-\frac{1}{2}\right) \int_{0}^{r} \frac{s^{\frac{1}{2}-H}\left(Z\left(Y_{r}^{\xi}\right)+h^{\xi}(r)-Z\left(Y_{s}^{\xi}\right)-h^{\xi}(s)\right)}{(r-s)^{H+\frac{1}{2}}} \mathrm{~d} s \\
& =: I_{3}(r)+I_{4}(r) \text {. }
\end{aligned}
$$

Next, we give the estimates for $I_{i}(r), i=3,4$, respectively. (H1) and (H2) yields that

$$
\begin{aligned}
\left|I_{3}(r)\right| & \leq\left[1+C_{0}\left(H-\frac{1}{2}\right)\right] \frac{r^{\frac{1}{2}-H}}{\Gamma\left(\frac{3}{2}-H\right)}\left(\left\|\widehat{\sigma}^{-1}\right\|\left|b\left(Y^{\xi}(r)\right)-b\left(Y^{\xi}\left(r_{\delta}\right)\right)\right|+\left\|Z\left(Y_{r}^{\xi}\right)-Z\left(\widehat{Y}_{r}^{\xi}\right)\right\|\right) \\
& \leq\left[1+C_{0}\left(H-\frac{1}{2}\right)\right] \frac{r^{\frac{1}{2}-H}}{\Gamma\left(\frac{3}{2}-H\right)}\left(L_{1}\left\|\widehat{\sigma}^{-1}\right\|\left\|Y^{\xi}\right\|_{0, r, \beta} \delta^{\beta}+L_{2}\left\|Y_{r}^{\xi}-\widehat{Y}_{r}^{\xi}\right\|_{\infty}^{\alpha}\right) \\
& \leq \frac{\left[1+C_{0}\left(H-\frac{1}{2}\right)\right] r^{\frac{1}{2}-H}}{\Gamma\left(\frac{3}{2}-H\right)}\left(L_{1}\left\|\widehat{\sigma}^{-1}\right\|\left\|Y^{\xi}\right\|_{0, r, \beta} \delta^{\beta}+L_{2}\left\|Y^{\xi}\right\|_{0, r, \beta}^{\alpha} \delta^{\alpha \beta}\right)
\end{aligned}
$$

Moreover, we have

$$
\begin{aligned}
\left|I_{4}(r)\right| \leq & \frac{r^{H-\frac{1}{2}}}{\Gamma\left(\frac{3}{2}-H\right)}\left(H-\frac{1}{2}\right) \int_{0}^{r} \frac{s^{\frac{1}{2}-H}\left(Z\left(Y_{r}^{\xi}\right)+h^{\xi}(r)-Z\left(Y_{s}^{\xi}\right)-h^{\xi}(s)\right)}{(r-s)^{H+\frac{1}{2}}} \mathrm{~d} s \\
\leq & \frac{r^{H-\frac{1}{2}}}{\Gamma\left(\frac{3}{2}-H\right)}\left(H-\frac{1}{2}\right)\left\|\widehat{\sigma}^{-1}\right\| \int_{0}^{r} \frac{s^{1 / 2-H}\left|b\left(Y^{\xi}(r)\right)-b\left(Y^{\xi}\left(r_{\delta}\right)\right)-\left(b\left(Y^{\xi}(s)\right)-b\left(Y^{\xi}\left(s_{\delta}\right)\right)\right)\right|}{(r-s)^{H+1 / 2}} \mathrm{~d} s \\
& +\frac{r^{H-\frac{1}{2}}}{\Gamma\left(\frac{3}{2}-H\right)}\left(H-\frac{1}{2}\right) \int_{0}^{r} \frac{s^{1 / 2-H}\left\|Z\left(Y_{r}^{\xi}\right)-Z\left(\widehat{Y}_{r}^{\xi}\right)-\left(Z\left(Y_{s}^{\xi}\right)-Z\left(\widehat{Y}_{s}^{\xi}\right)\right)\right\|}{(r-s)^{H+1 / 2}} \mathrm{~d} s \\
= & I_{41}(r)+I_{42}(r) .
\end{aligned}
$$

In the same way to estimate $I_{1}$ in the proof of Lemma 5.2, we have

$$
I_{41}(r)
$$




$$
\begin{aligned}
\leq & 2 \frac{r^{H-\frac{1}{2}}}{\Gamma\left(\frac{3}{2}-H\right)}\left(H-\frac{1}{2}\right) L_{1}\left\|\widehat{\sigma}^{-1}\right\|\left\|Y^{\xi}\right\|_{0, r, \beta}\left\{\left[\frac{\delta^{\beta+1 / 2-H}}{(2 \beta+1-2 H) r_{\delta}^{H-1 / 2}}+\frac{\delta^{\beta}\left(r-r_{\delta}\right)^{\frac{1}{2}-H}}{\left(r_{\delta} / 2\right)^{H-1 / 2}(H-1 / 2)}\right.\right. \\
& \left.\left.+\frac{\delta^{\beta}\left(\frac{r_{\delta}}{2}\right)^{\frac{3}{2}-H}}{\left(r-r_{\delta} / 2\right)^{H+1 / 2}\left(\frac{3}{2}-H\right)}\right] \mathbb{1}_{[r \geq \delta]}+\frac{1}{2} \mathcal{B}\left(\beta+\frac{1}{2}-H, \frac{3}{2}-H\right) r^{\beta+1-2 H} \mathbb{1}_{[0 \leq r<\delta]}\right\} . \quad
\end{aligned}
$$

On the other hand, it follows from (H2) that

$$
\begin{aligned}
\left\|Z\left(Y_{r}^{\xi}\right)-Z\left(\widehat{Y}_{r}^{\xi}\right)-\left(Z\left(Y_{s}^{\xi}\right)-Z\left(\widehat{Y}_{s}^{\xi}\right)\right)\right\| & \leq L_{2}\left\|Y_{r}^{\xi}-\widehat{Y}_{r}^{\xi}\right\|_{\infty}^{\alpha}+L_{2}\left\|Y_{s}^{\xi}-\widehat{Y}_{s}^{\xi}\right\|_{\infty}^{\alpha} \\
& \leq 2 L_{2}\|Y\|_{-\tau, r, \beta \wedge \theta}^{\alpha} \delta^{\alpha(\beta \wedge \theta)}
\end{aligned}
$$

and

$$
\begin{aligned}
& \left\|Z\left(Y_{r}^{\xi}\right)-Z\left(\widehat{Y}_{r}^{\xi}\right)-\left(Z\left(Y_{s}^{\xi}\right)-Z\left(\widehat{Y}_{s}^{\xi}\right)\right)\right\| \\
& \quad \leq L_{2}\left\|Y_{r}^{\xi}-Y_{s}^{\xi}\right\|_{\infty}^{\alpha}+L_{2}\left\|\widehat{Y}_{r}^{\xi}-\widehat{Y}_{s}^{\xi}\right\|_{\infty}^{\alpha} \\
& \quad \leq L_{2}\|Y\|_{-\tau, r, \beta \wedge \theta}^{\alpha}|r-s|^{\alpha(\beta \wedge \theta)}+L_{2}\|Y\|_{-\tau, r, \beta \wedge \theta}^{\alpha}\left(|r-s|^{\alpha(\beta \wedge \theta)} \vee\left|r_{\delta}-s_{\delta}\right|^{\alpha(\beta \wedge \theta)}\right) \\
& \quad=L_{2}\|Y\|_{-\tau, r, \beta \wedge \theta}^{\alpha}\left(|r-s|^{\alpha(\beta \wedge \theta)}+|r-s|^{\alpha(\beta \wedge \theta)} \vee\left|r_{\delta}-s_{\delta}\right|^{\alpha(\beta \wedge \theta)}\right) .
\end{aligned}
$$

Combining these two upper bounds together, we have

$$
\begin{aligned}
& \left\|Z\left(Y_{r}^{\xi}\right)-Z\left(\widehat{Y}_{r}^{\xi}\right)-\left(Z\left(Y_{s}^{\xi}\right)-Z\left(\widehat{Y}_{s}^{\xi}\right)\right)\right\| \\
& \quad \leq 2 L_{2}\left\|Y^{\xi}\right\|_{-\tau, r, \beta \wedge \theta}^{\alpha}\left(\delta^{\alpha(\beta \wedge \theta)} \wedge \frac{|r-s|^{\alpha(\beta \wedge \theta)}+|r-s|^{\alpha(\beta \wedge \theta)} \vee\left|r_{\delta}-s_{\delta}\right|^{\alpha(\beta \wedge \theta)}}{2}\right) .
\end{aligned}
$$

Since for $r \geq \delta$,

$$
\delta^{\alpha(\beta \wedge \theta)} \wedge \frac{|r-s|^{\alpha(\beta \wedge \theta)}+|r-s|^{\alpha(\beta \wedge \theta)} \vee\left|r_{\delta}-s_{\delta}\right|^{\alpha(\beta \wedge \theta)}}{2}=\delta^{\alpha(\beta \wedge \theta)}, s \in[0, r-\delta],
$$

we get

$$
\begin{aligned}
\int_{0}^{r_{\delta}} & \frac{\delta^{\alpha(\beta \wedge \theta)} \wedge \frac{|r-s|^{\alpha(\beta \wedge \theta)}+|r-s|^{\alpha(\beta \wedge \theta)} \vee\left|r_{\delta}-s_{\delta}\right|^{\alpha(\beta \wedge \theta)}}{2}}{(r-s)^{H+1 / 2} s^{H-1 / 2}} \mathrm{~d} s \\
\quad & \int_{0}^{r_{\delta}} \frac{\delta^{\alpha(\beta \wedge \theta)}}{(r-s)^{H+1 / 2} s^{H-1 / 2}} \mathrm{~d} s \\
& \leq \frac{\delta^{\alpha(\beta \wedge \theta)}\left(r-r_{\delta}\right)^{\frac{1}{2}-H}}{\left(r_{\delta} / 2\right)^{H-1 / 2}(H-1 / 2)}+\frac{\delta^{\alpha(\beta \wedge \theta)}\left(\frac{r_{\delta}}{2}\right)^{\frac{3}{2}-H}}{\left(r-r_{\delta} / 2\right)^{H+1 / 2}\left(\frac{3}{2}-H\right)}
\end{aligned}
$$

and

$$
\int_{r_{\delta}}^{r} \frac{\delta^{\alpha(\beta \wedge \theta)} \wedge \frac{|r-s|^{\alpha(\beta \wedge \theta)}+|r-s|^{\alpha(\beta \wedge \theta)} \vee\left|r_{\delta}-s_{\delta}\right|^{\alpha(\beta \wedge \theta)}}{2}}{(r-s)^{H+1 / 2} s^{H-1 / 2}} \mathrm{~d} s
$$




$$
\begin{aligned}
& =\int_{r_{\delta}}^{r} \frac{(r-s)^{\alpha(\beta \wedge \theta)}}{(r-s)^{H+1 / 2} s^{H-1 / 2}} \mathrm{~d} s \\
& \leq \frac{r_{\delta}^{\frac{1}{2}-H}\left(r-r_{\delta}\right)^{\alpha(\beta \wedge \theta)+\frac{1}{2}-H}}{\alpha(\beta \wedge \theta)+\frac{1}{2}-H} \\
& \leq \frac{2 r_{\delta}^{\frac{1}{2}-H} \delta^{\alpha(\beta \wedge \theta)+\frac{1}{2}-H}}{2 \alpha(\beta \wedge \theta)+1-2 H} .
\end{aligned}
$$

Thus,

$$
\begin{aligned}
& \left|I_{42}(r)\right| \leq \frac{2 L_{2}\left(H-\frac{1}{2}\right) r^{H-\frac{1}{2}}}{\Gamma\left(\frac{3}{2}-H\right)}\left\|Y^{\xi}\right\|_{-\tau, r, \beta \wedge \theta}^{\alpha}\left\{\left(\frac{\delta^{\alpha(\beta \wedge \theta)}\left(r-r_{\delta}\right)^{\frac{1}{2}-H}}{\left(r_{\delta} / 2\right)^{H-1 / 2}(H-1 / 2)}+\frac{\delta^{\alpha(\beta \wedge \theta)}\left(\frac{r_{\delta}}{2}\right)^{\frac{3}{2}-H}}{\left(r-r_{\delta} / 2\right)^{H+1 / 2}\left(\frac{3}{2}-H\right)}\right.\right. \\
& \left.\left.+\frac{2 r_{\delta}^{\frac{1}{2}-H} \delta^{\alpha(\beta \wedge \theta)+\frac{1}{2}-H}}{2 \alpha(\beta \wedge \theta)+1-2 H}\right) \mathbb{1}[r \geq \delta]+\mathcal{B}\left(\frac{3}{2}-H, \alpha(\beta \wedge \theta)+\frac{1}{2}-H\right) r^{\alpha(\beta \wedge \theta)+\frac{1}{2}-H} \mathbb{1}_{[0 \leq r<\delta]}\right\} .
\end{aligned}
$$

Substituting (5.14), (5.15), (5.12) and (5.13) into (5.11), we arrive at

$$
\begin{aligned}
\mid K_{H}^{-1} & \left(\int_{0}\left(Z\left(Y_{s}^{\xi}\right)+h^{\xi}(s)\right) \mathrm{d} s\right)(r) \mid \\
& \leq\left[1+C_{0}\left(H-\frac{1}{2}\right)\right] \frac{r^{\frac{1}{2}-H}}{\Gamma\left(\frac{3}{2}-H\right)}\left(L_{1}\left\|\widehat{\sigma}^{-1}\right\|\left\|Y^{\xi}\right\|_{0, r, \beta} \delta^{\beta}+L_{2}\left\|Y^{\xi}\right\|_{0, r, \beta}^{\alpha} \delta^{\alpha \beta}\right) \\
& +\frac{r^{H-1 / 2}(2 H-1)}{\Gamma\left(\frac{3}{2}-H\right)}\left\{L _ { 1 } \| \widehat { \sigma } ^ { - 1 } \| \| Y ^ { \xi } \| _ { 0 , r , \beta } \left[\frac{\delta^{\beta+1 / 2-H}}{(2 \beta+1-2 H) r_{\delta}^{H-1 / 2}}+\frac{\delta^{\beta}\left(r-r_{\delta}\right)^{\frac{1}{2}-H}}{\left(r_{\delta} / 2\right)^{H-1 / 2}(H-1 / 2)}\right.\right. \\
& \left.+\frac{\delta^{\beta}\left(\frac{r_{\delta}}{2}\right)^{\frac{3}{2}-H}}{\left(r-r_{\delta} / 2\right)^{H+1 / 2}\left(\frac{3}{2}-H\right)}\right]+L_{2}\left\|Y^{\xi}\right\|_{-\tau, r,(\beta \wedge \theta)}^{\alpha}\left[\frac{\delta^{\alpha(\beta \wedge \theta)}\left(r-r_{\delta}\right)^{\frac{1}{2}-H}}{\left(r_{\delta} / 2\right)^{H-1 / 2}(H-1 / 2)}\right. \\
& \left.\left.+\frac{\delta^{\alpha(\beta \wedge \theta)}\left(\frac{r_{\delta}}{2}\right)^{\frac{3}{2}-H}}{\left(r-r_{\delta} / 2\right)^{H+1 / 2}\left(\frac{3}{2}-H\right)}+\frac{2 r_{\delta}^{\frac{1}{2}-H} \delta^{\alpha(\beta \wedge \theta)+\frac{1}{2}-H}}{2 \alpha(\beta \wedge \theta)+1-2 H}\right]\right\} \mathbb{1}_{[r \geq \delta]} \\
& +\frac{r^{\frac{1}{2}-H}(2 H-1)}{\Gamma\left(\frac{3}{2}-H\right)}\left\{\frac{L_{1}\left\|\widehat{\sigma}^{-1}\right\|}{2} \mathcal{B}\left(\frac{3}{2}-H, \frac{1}{2}+\beta-H\right)\left\|Y^{\xi}\right\|_{0, r, \beta} r^{\beta+1-2 H}\right. \\
& \left.+L_{2} \mathcal{B}\left(\frac{3}{2}-H, \frac{1}{2}+\alpha(\beta \wedge \theta)-H\right)\left\|Y^{\xi}\right\|_{-\tau, r, \beta \wedge \theta}^{\alpha} r^{\alpha(\beta \wedge \theta)+\frac{1}{2}-H}\right\} \mathbb{1}_{[0 \leq r<\delta]} .
\end{aligned}
$$

By the B-D-G inequality,

$$
\begin{aligned}
\Theta_{2}(t) & \leq C_{T}\left(\mathbb{E}\left(\int_{0}^{T}\left|K_{H}^{-1}\left(\int_{0}\left(Z\left(Y_{s}^{\xi}\right)+h^{\xi}(s)\right) \mathrm{d} s\right)(r)\right|^{2} \mathrm{~d} r\right)^{\frac{q}{(q-1) 2}}\right)^{\frac{q-1}{q}} \\
& \leq C_{T} \delta^{\alpha(\beta \wedge \theta)+\frac{1}{2}-H} .
\end{aligned}
$$


For $\Theta_{3}$, it follows from Hölder's inequality and (5.16) that

$$
\begin{aligned}
\Theta_{3}(t) \leq & \frac{1}{2}\left(\mathbb{E}\left(\int_{0}^{T}\left|K_{H}^{-1}\left(\int_{0}^{.}\left(Z\left(Y_{s}^{\xi}\right)-h^{\xi}(s)\right) \mathrm{d} s\right)(r)\right|^{2} \mathrm{~d} r\right)^{\frac{q}{q-1}}\right)^{\frac{q-1}{2 q}} \\
& \times\left(\mathbb{E}\left(\int_{0}^{T}\left|K_{H}^{-1}\left(\int_{0}^{.}\left(Z\left(Y_{s}^{\xi}\right)+h^{\xi}(s)\right) \mathrm{d} s\right)(r)\right|^{2} \mathrm{~d} r\right)^{\frac{q}{q-1}}\right)^{\frac{q-1}{2 q}} \\
\leq & C_{T} \delta^{\alpha(\beta \wedge \theta)+\frac{1}{2}-H}\left(\mathbb{E}\left(\int_{0}^{T}\left|K_{H}^{-1}\left(\int_{0}^{.}\left(Z\left(Y_{s}^{\xi}\right)-h^{\xi}(s)\right) \mathrm{d} s\right)(r)\right|^{2} \mathrm{~d} r\right)^{\frac{q}{q-1}}\right)^{\frac{q-1}{2 q}} .
\end{aligned}
$$

Since

$$
\begin{aligned}
\int_{0}^{T}\left|K_{H}^{-1}\left(\int_{0}\left(Z\left(Y_{s}^{\xi}\right)-h^{\xi}(s)\right) \mathrm{d} s\right)\right|^{2}(r) \mathrm{d} r \leq 2 & \int_{0}^{T}\left|K_{H}^{-1}\left(\int_{0}^{\cdot}\left(Z\left(Y_{s}^{\xi}\right)+h^{\xi}(s)\right) \mathrm{d} s\right)\right|^{2}(r) \mathrm{d} r \\
& +2 \int_{0}^{T}\left|K_{H}^{-1}\left(\int_{0}^{\cdot} h^{\xi}(s) \mathrm{d} s\right)\right|^{2}(r) \mathrm{d} r
\end{aligned}
$$

it follows from (5.16) and (5.8) that

$$
\left(\mathbb{E}\left(\int_{0}^{T}\left|K_{H}^{-1}\left(\int_{0}^{.}\left(Z\left(Y_{s}^{\xi}\right)-h^{\xi}(s)\right) \mathrm{d} s\right)\right|^{2}(r) \mathrm{d} r\right)^{\frac{q}{q-1}}\right)^{\frac{q-1}{2 q}}<\infty .
$$

Hence,

$$
\Theta_{3}(t) \leq C_{T} \delta^{\alpha(\beta \wedge \theta)+\frac{1}{2}-H}
$$

Finally, the desired assertion is established from (5.9) and the estimates of $\Theta_{i}(t), i=$ $1,2,3$.

\section{References}

[1] Bao, J., Shao, J., Weak convergence of path-dependent SDEs with irregular coefficients, arxiv:1809.03088

[2] Boufoussi, B., Hajji, S., Functional differential equations driven by a fractional Brownian motion. Comput. Math. Appl. 62 (2011), no. 2, 746-754.

[3] Coutin, L., Qian, Z., Stochastic analysis, rough path analysis and fractional Brownian motions. Probab. Theory Related Fields 122 (2002), no. 1, 108-140.

[4] Decreusefond, L., Üstünel, A.S., Stoshastic analysis of the functional brownian motion. Potential Anal. 10(1998), 177-214. 
[5] Fan, X., Zhang, S., On SDEs driven by fractional Brownian motions with irregular drifts. arxiv:1810.01669

[6] Ferrante, M., Rovira, C., Stochastic delay differential equations driven by fractional Brownian motion with Hurst parameter $H>\frac{1}{2}$. Bernoulli 12 (2006), no. 1, 85-100.

[7] Hu, Y., Liu, Y., Nualart, D., Rate of convergence and asymptotic error distribution of Euler approximation schemes for fractional diffusions. Ann. Appl. Probab. 26 (2016), no. $2,1147-1207$.

[8] Hu, Y., Nualart, D., Differential equations driven by Hölder continuous functions of order greater than $\frac{1}{2}$. Stochastic analysis and applications, 399-413, Abel Symp., 2, Springer, Berlin, 2007.

[9] Hu, Y., Nualart, D., Song, X., A singular stochastic differential equation driven by fractional Brownian motion. Statist. Probab. Lett. 78 (2008), no. 14, 2075-2085.

[10] Kohatsu-Higaa, A., Lejay, A., Yasuda, K., Weak rate of convergence of the EulerMaruyama scheme for stochastic differential equations with non-regular drift, Journal of Computational and Applied Mathematics 326 (2017), 138-158.

[11] Konakov, V., Menozzi, S., Weak error for the Euler scheme approximation of diffusions with non-smooth coefficients, Electron. J. Probab. 22 (2017), no. 46, 1-47.

[12] Liu, J., Yan, L., On a nonlinear stochastic pseudo-differential equation driven by fractional noise. Stoch. Dyn. 18 (2018), no. 1, 1850002.

[13] Lyons, T., Differential equations driven by rough signals. I. An extension of an inequality of L. C. Young. Math. Res. Lett. 1 (1994), no. 4, 451-464.

[14] Meerschaert, M. M., Wang, W. and Xiao, Y., Fernique-type inequalities and moduli of continuity for anisotropic Gaussian random fields, Trans Am Math Soc. ; 2013(365): 1081-1107.

[15] Mishura, Y., Shevchenko, G., The rate of convergence for Euler approximations of solutions of stochastic differential equations driven by fractional Brownian motion. Stochastics. 80 (2008), 489-511.

[16] Mishura, Y., Shevchenko, G., Rate of convergence of Euler approximations of solution to mixed stochastic differential equation involving Brownian motion and fractional Brownian motion. Random Oper. Stoch. Equ. 19 (2011), no. 4, 387-406.

[17] Mishura, Y., Shevchenko, G., Existence and uniqueness of the solution of stochastic differential equation involving Wiener process and fractional Brownian motion with Hurst index $H>1 / 2$. Comm. Statist. Theory Methods 40 (2011), no. 19-20, 3492-3508. 
[18] Mishura, Y., Shevchenko, G., Mixed stochastic differential equations with long-range dependence: Existence, uniqueness and convergence of solutions. Comput. Math. Appl. 64 (2012), no. 10, 3217-3227.

[19] Mishura, Y., Shalaiko, T., Shevchenko, G., Convergence of solutions of mixed stochastic delay differential equations with applications, Applied Mathematics and Computation.257(2015), 487-497.

[20] Neuenkirch, A., Optimal approximation of SDE's with additive fractional noise. J. Complexity 22 (2006), no. 4, 459-474.

[21] Neuenkirch, A., Nourdin, I., Tindel, S., Delay equations driven by rough paths. Electron. J. Probab. 13 (2008), no. 67, 2031-2068.

[22] Ngo, H.-L., Taguchi, Dai, Approximation for non-smooth functionals of stochastic differential equations with irregular drift, J. Math. Anal. Appl. 457 (2018) 361-388.

[23] Nualart, D., The malliavin calculus and related topics, Second edition, Springer-Verlag, Berlin, 2006.

[24] Nualart, D., Ouknine, Y., Regularization of differential equations by fractional noise. Stochastic Process. Appl. 102 (2002), no. 1, 103-116.

[25] Nualart, D., Răşcanu, A., Differential equations driven by fractional Brownian motion. Collect. Math. 53 (2002), no. 1, 55-81.

[26] Saussereau, B., Transportation inequalities for stochastic differential equations driven by a fractional Brownian motion. Bernoulli 18 (2012), no. 1, 1-23.

[27] Samko, S.G., Kilbas, A.A., Marichev, O.I., Fractional Integrals and Derivatives, Theory and Applications, Gordon and Breach Science Publishers, Yvendon, 1993.

[28] Shklyar, S., Shevchenko, G., Mishura, Y., Doroshenko, V., Banna, O., Approximation of fractional Brownian motion by martingales. Methodol. Comput. Appl. Probab. 16 (2014), no. 3, 539-560.

[29] Wang, F.Y., Estimates for invariant probability measures of degenerate SPDEs with singular and path-dependent drifts. Probab. Theory Relat. Fields. 172 (2018), 11811214

[30] Young, L. C., An inequality of the Hölder type, connected with Stieltjes integration. Acta Math. 67 (1936), no. 1, 251-282.

[31] Zähle, M. Integration with respect to fractal functions and stochastic calculus. I. Probab. Theory Related Fields 111 (1998), no. 3, 333-374. 